\title{
Is the EMI model a QFT? An inquiry on the space of allowed entropy functions
}

\author{
César A. Agón, Pablo Bueno and Horacio Casini \\ Instituto Balseiro, Centro Atómico Bariloche, \\ 8400-S.C. de Bariloche, Río Negro, Argentina \\ E-mail: cesaragon1@gmail.com, pablo.bueno@cab.cnea.gov.ar, \\ casini@cab.cnea.gov.ar
}

ABstract: The mutual information $I(A, B)$ of pairs of spatially separated regions satisfies, for any $d$-dimensional CFT, a set of structural physical properties such as positivity, monotonicity, clustering, or Poincaré invariance, among others. If one imposes the extra requirement that $I(A, B)$ is extensive as a function of its arguments (so that the tripartite information vanishes for any set of regions, $\left.I_{3}(A, B, C) \equiv 0\right)$, a closed geometric formula involving integrals over $\partial A$ and $\partial B$ can be obtained. We explore whether this "Extensive Mutual Information" model (EMI), which in fact describes a free fermion in $d=2$, may similarly correspond to an actual CFT in general dimensions. Using the long-distance behavior of $I_{\mathrm{EMI}}(A, B)$ we show that, if it did, it would necessarily include a free fermion, but also that additional operators would have to be present in the model. Remarkably, we find that $I_{\mathrm{EMI}}(A, B)$ for two arbitrarily boosted spheres in general $d$ exactly matches the result for the free fermion current conformal block $G_{\Delta=(d-1), J=1}^{d}$. On the other hand, a detailed analysis of the subleading contribution in the long-distance regime rules out the possibility that the EMI formula represents the mutual information of any actual CFT or even any limit of CFTs. These results make manifest the incompleteness of the set of known constraints required to describe the space of allowed entropy functions in QFT.

KEywords: Conformal Field Theory, Field Theories in Higher Dimensions

ARXiv EPrint: 2105.11464 


\section{Contents}

1 Introduction 1

1.1 Summary of results 4

2 EMI contains a free fermion $\quad 5$

2.1 Two dimensions 7

$\begin{array}{ll}2.2 & \text { Not a free fermion for } d>2\end{array}$

3 Current conformal block equals EMI for spherical regions 11

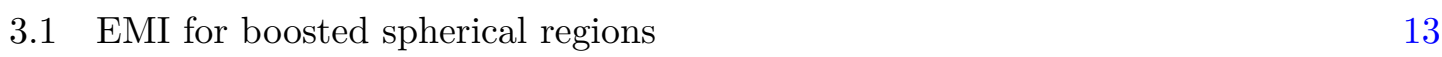

$\begin{array}{lll}3.1 .1 & \text { Long distance } & 17\end{array}$

$\begin{array}{lll}3.1 .2 & \text { Zero relative boost } & 19\end{array}$

$\begin{array}{lll}\text { 3.1.3 General case for particular dimensions } & 21\end{array}$

4 Free fermion at long distances for arbitrary regions $\quad 24$

5 Is the EMI the mutual information of a CFT? 27

6 Can EMI represent a limit of CFTs? 28

$\begin{array}{lll}\text { 6.1 Further analysis of the free fermion MI for spheres } & 29\end{array}$

$\begin{array}{lll}7 & \text { Discussion } & 33\end{array}$

A EE universal coefficients for spheres and strips for the EMI 36

$\begin{array}{ll}\text { B OPE blocks and the EMI } & 38\end{array}$

C Conformal blocks for conserved currents $\quad 40$

D Coefficient of the first subleading term in the MI for a free fermion 42

\section{Introduction}

In its most straightforward formulation, quantum field theory (QFT) is defined in terms of a set of field operators acting on Hilbert space. It is well known that in the relativistic case it can be equivalently defined through correlation functions, i.e., vacuum expectation values of products of fields [1]. Hence, the vacuum state and its relation to the local operator content hold the full information of the theory. The step of moving from operators to numbers turns out to be very fruitful in simplifying the mathematical description and providing resources for the elaboration of specific models, e.g., the path integral formulation for Euclidean functions. 
Another formulation of QFT is based on the assignation of operator algebras to open regions of spacetime, rather than field operators at a point [2]. This is conceptually very natural and can be thought of as a "basis independent" formulation since the same algebras may be generated by many different sets of fields. One inconvenience is that the mathematical description gets more complicated, involving the theory of von Neumann algebras. In this sense, to simplify the description of models in this context, and in analogy to the field operator description, a natural idea is to assign numbers to these algebras using the vacuum state. These numbers could only represent statistical measures of vacuum fluctuations for the different regions.

Among the different possible statistical measures, the entanglement entropy (EE) is highlighted by its simplicity, powerful properties, and connections with statistical mechanics, as well as its important role in holographic models. EE is a function of the geometry of the regions - a "statistical correlator" — which can be universally defined for any QFT. The entropy has ultraviolet divergences, but this is unimportant since a simple combination of entropies for two non-intersecting regions $A, B$, called mutual information (MI),

$$
I(A, B) \equiv S(A)+S(B)-S(A B)
$$

is finite and well defined, and arguably contains the full universal information of the entropy.

A natural question is whether the MI function contains enough information to uniquely determine the models. The answer seems to be positive. For example, it is known that for a CFT the MI for faraway regions has a series expansion in the distance from where the conformal dimensions of primary fields may be extracted $[3,4]$. The rest of the CFT data, the coefficient of the three point functions, may be obtained from the MI involving three or more distant regions [5].

A different but related question is: what are the rules for a function $I(A, B)$ to be admissible as the MI of a QFT? Surprisingly little is known about this important question. A simple list is the following. We recall that $I(A, B)$ is a function of causally closed, ${ }^{1}$ spatially separated regions $A, B$ in Minkowski space. Defining the tripartite information function

$$
I_{3}(A, B, C)=I_{3}(A, C, B) \equiv I(A, B)+I(A, C)-I(A, B C),
$$

calling $\Lambda$ to a Lorentz transformation, and taking $X, Y$, as regions with spatial boundary in a common null plane, we have

$$
\begin{aligned}
I(A, B) & \geq 0, & & \text { (positivity) } \\
B \subseteq C \Longrightarrow I(A, B) & \leq I(A, C), & & \text { (monotonicity) } \\
I(A, B)=I(B, A), \quad I_{3}(A, B, C) & =I_{3}(B, C, A), & & \text { (symmetry) } \\
I(A, B) & =I(\Lambda A+x, \Lambda B+x), & & \text { (Poincaré invariance) } \\
\lim _{|x| \rightarrow \infty} I(A, B+x) & =0, & & \text { (clustering) } \\
I(A, X)+I(A, Y) & \leq I(A, X \cap Y)+I(A, X \cup Y) . & & \text { (Markov property) }
\end{aligned}
$$

\footnotetext{
${ }^{1} \mathrm{~A}$ region is causally closed (or a "diamond-shaped set") if it is the set of all points spatial to another region.
} 
Positivity and monotonicity are properties of MI as a measure of correlations, monotonicity encoding strong subadditivity of the entropy [6]. The symmetry properties (1.5) tell that MI is in fact a combination of (undefined) single region functions as in (1.1). Poincaré invariance and clustering follow the same properties of ordinary correlators. The Markov property (1.8) expresses strong superadditivity of mutual information when two regions $X, Y$ have boundary on a null plane. This follows from the Markov property of entropy in a null plane and strong subadditivity [7]. Given Lorentz invariance and certain additional (natural) continuity assumptions, the entropy is Markovian on the null plane $[8,9]$, so (1.8) is probably an unavoidable consequence of (1.4), (1.5) and (1.6). This Markov property has been shown to imply unitarity bounds in [7].

Any QFT gives a solution to this set of axioms. But we also have solutions in dimension $d$ by dimensional reduction from theories in higher dimensions. To eliminate these and fix $d$-dimensional QFT we can further impose that for two disjoint regions $A, B$ sharing a planar boundary with normal $\eta$

$$
I(A, B+\epsilon \eta) \sim \epsilon^{-(d-2)}, \quad \epsilon \rightarrow 0 . \quad \text { (area law) }
$$

In studying what other properties are needed we can search for solutions to this set of properties and see if the result is physically sensible. If it is not, the hope is that it would give us a hint of what is missing. One striking difference with the case of operator correlations is the two inequalities (1.3), (1.4), instead of an infinite tower of inequalities. This is more so considering that Rényi entropies of integer index $n$ (the entropy is the limit $n \rightarrow 1$ ) do indeed obey an infinite tower of inequalities [10].

It is not easy to obtain solutions in a direct way. A notable example with a purely geometric solution is given by the holographic EE [11-15]. Another one is the extensive mutual information model (EMI) [16]. This last model simplifies the symmetry requirement by imposing

$$
I_{3}(A, B, C) \equiv 0 .
$$

According to the definition of $I_{3}$, eq. (1.2), this implies an extensivity or additivity of the mutual information as a function of its arguments, and hence the name of the model. If we further impose conformal invariance there is a unique solution (except for a global multiplicative constant). This is given by

$$
I_{\mathrm{EMI}}(A, B)=2 \kappa_{(d)} \int_{\Sigma_{A}} \mathrm{~d} \sigma_{A} \int_{\Sigma_{B}} \mathrm{~d} \sigma_{B} \eta_{A}^{\mu}\left(x_{A}\right) \eta_{B}^{\nu}\left(x_{B}\right)\left(\partial_{\mu} \partial_{\nu}-g_{\mu \nu} \partial^{2}\right)\left|x_{A}-x_{B}\right|^{-2(d-2)},
$$

where $\Sigma_{A}, \Sigma_{B}$, are any Cauchy surfaces for the causal regions $A, B ; \eta_{A}, \eta_{B}$, are the unit normals to these surfaces, and $\kappa_{(d)}$ is a positive constant. The integrand is a conserved current in both indices, which guarantees the result to be independent of the Cauchy surface. In fact, this expression is equivalent to one where the integration is only on the spatial boundaries $\partial A, \partial B$, of the regions:

$$
I_{\mathrm{EMI}}(A, B)=2 \kappa_{(d)} \int_{\partial A} \mathrm{~d} \sigma_{A} \int_{\partial B} \mathrm{~d} \sigma_{B} \frac{\left(n_{A} \cdot n_{B}\right)\left(\bar{n}_{A} \cdot \bar{n}_{B}\right)-\left(n_{A} \cdot \bar{n}_{B}\right)\left(\bar{n}_{A} \cdot n_{B}\right)}{\left|x_{A}-x_{B}\right|^{2(d-2)}},
$$


where $\bar{n}_{A}$ and $n_{A}$ are unit vectors orthogonal to the surface and to each other, $n_{A} \cdot \bar{n}_{A}=$ $0 .^{2} \quad$ For fixed time slices, one can choose $n_{A}=n_{B}=\hat{t}$ and the integrand reduces to $-\left(\bar{n}_{A} \cdot \bar{n}_{B}\right) /\left|x_{A}-x_{B}\right|^{2(d-2)}$.

The EMI model gives physically reasonable results, and has been mainly used to explore the expected behavior of the EE in different geometric situations [17-21]. In general, the results are quite similar to the free fermion ones, and the EMI model indeed coincides with a free massless fermion in $d=2$ [22]. Another way to arrive at the EMI formula is to assume the Rényi twist operators are exponentials of free fields [23]. This automatically gives the bilinear form on the boundaries as in (1.12) from the expectation values of the Rényi operators.

In this paper, we investigate if the EMI model gives the mutual information of some CFT in $d \geq 3$. In other words, we test the consistency of imposing eq. (1.10) in QFT. To understand the possible theory behind (1.12), we use known relations between the behavior of mutual information at long distances and the operator content of CFTs. We find that no possible CFT or limit of CFTs is consistent with the extensivity condition. This result makes manifest that the list of known constraints or axioms satisfied by the MI of spatially separated regions in QFT is incomplete.

A more detailed list of our findings can be found next.

\subsection{Summary of results}

In section 2, we show that in case the EMI formula describes the MI of an actual CFT, this necessarily contains a free fermion. This is done by considering the leading contribution to the long-distance behavior of the EMI in the case of two boosted spheres, which reveals the same tensorial structure as in the case of a free fermion. Then, we review the fact that the EMI indeed coincides with a free fermion in $d=2$. Moving to $d \geq 3$, we perform various comparisons between universal coefficients characterizing the EMI and the free fermion theories and show that these differ for both models, the discrepancy growing with $d$.

In section 3 we compute the EMI result in the case of two spatially separated and arbitrarily boosted spheres. For a general CFT, such MI can be written as a linear combination of the conformal blocks associated to each primary operator in the replica theory, $I(A, B)=\sum_{\Delta, J} b_{\Delta, J} G_{\Delta, J}^{d}(u, v)$, where $u, v$ are the relevant conformal cross-ratios. Using the fact that a free fermion controls the leading piece, the EMI is argued to contain a leading piece associated to such field with $\Delta=d-1$ and $J=1$. Interestingly, we find that this is actually the full result for the EMI model, i.e.,

$$
I_{\mathrm{EMI}}(A, B)=b_{d-1,1}^{(\text {ferm })} G_{d-1,1}^{d}(u, v) .
$$

In other words, the EMI result for two spheres turns out to coincide with the conformal block associated to the current operator $J_{\mu}=\bar{\psi} \gamma_{\mu} \psi$. In passing, we obtain some new explicit formulas for $G_{d-1,1}^{d}(u, v)$ for different values of $d$, some of them not available in the literature.

\footnotetext{
${ }^{2}$ The factor 2 in this formula is conventional, and devised to get the same formula with $\partial A=\partial B$ for the entropy $S(A)$, with $\kappa_{(d)}$ as a coefficient.
} 
In section 4 we test the hypothesis that the EMI may coincide with the free fermion at long distances for arbitrary regions. Considering rectangular regions for $d=3$ fermions in the lattice, we show that this is not the case. An analytic general- $d$ argument in the same direction is provided using regions with very thin parallel sides.

In section 5 we use the results of the previous sections to argue that the EMI cannot describe the MI of any CFT. This follows from the fact that the free fermion present in the putative CFT describing the EMI decouples - based on standard QFT observations - from the rest of the theory. This would imply that the long-distance behavior of the EMI should match the free fermion one for arbitrary regions, which contradicts the results of the previous section.

In section 6 we consider the possibility that the EMI may correspond to some limit of CFTs, something that cannot be discarded from our previous analysis. For instance, if we tried to extract the operator content of the dual holographic theory using the longdistance result for the MI produced by the Ryu-Takayanagi formula - corresponding to the large- $N$ limit - we would wrongly conclude that the theory is empty of operators. Considering the structure of the subleading piece produced by the free fermion in the EMI expansion for spheres separated a long distance and the possible ways in which this could be compensated by extra fields, we can prove that — as opposed to the Ryu-Takayanagi formula - the EMI cannot correspond to a limit of theories either.

In section 7 we make some further comments regarding the interpretation of the EMI model and the possible ways in which the set of known axioms for the MI in QFT may be enhanced.

Appendix A contains a calculation of the entanglement entropy universal coefficients for sphere and strip regions in general dimensions for the EMI model. In appendix B we provide a proof of the equivalence between the EMI and the conformal block $G_{d-1,1}^{d}(u, v)$. Appendix $\mathrm{C}$ contains some new formulas for the conformal blocks in the cases corresponding to conserved currents, $\Delta=J+d-2$. Finally, appendix D includes a calculation of the coefficient appearing in the subleading contribution to the MI of a free fermion for large separations.

\section{EMI contains a free fermion}

In this section, we show that, if the EMI is a CFT in general dimensions, this necessarily contains a free fermion as the lowest dimensional operator. We then review the known fact that in $d=2$ the EMI indeed coincides with the free fermion theory and how this match does not hold in higher dimensions.

The leading term in the expansion of MI for long separating distance $L$ between $A$ and $B$ is known to be dominated by the lowest dimensional primary field (or fields) of the CFT [3]. The general expression for this leading term is

$$
I(A, B) \sim C(A, B)\left(\frac{R_{A} R_{B}}{L^{2}}\right)^{2 \Delta},
$$

where $\Delta$ is the lowest dimension of the theory, $R_{A}, R_{B}$, are some typical length scales of the regions, and $C(A, B)$ is a dimensionless coefficient. In general, the form of this coefficient 
depends on the full operator content of the theory. A formula for $C(A, B)$ in terms of the modular flows of the regions $A$ and $B$ has been recently derived in [7]. For the special case where the two entangling surfaces are spheres, $\partial A=\partial B=\mathbb{S}^{d-2}$, this modular flow is universal and geometric in a CFT [24]. As a consequence of that, a closed expression for the coefficient is known in the case of spheres. This was first obtained for scalar fields in [4]. Taking $R_{A}$ and $R_{B}$ in (2.1) as their radii, such coefficient is independent of the spheres orientations in spacetime and reduces to a function of the conformal dimension. We have [4]

$$
I_{\text {scal }}(A, B)=c(\Delta)\left(\frac{R_{A} R_{B}}{L^{2}}\right)^{2 \Delta}+\ldots, \quad \text { where } \quad c(\Delta) \equiv \frac{\sqrt{\pi} \Gamma(2 \Delta+1)}{4 \Gamma\left(2 \Delta+\frac{3}{2}\right)} .
$$

Because the geometric form of the modular flow is independent of the spacetime dimension $d$, this coefficient only depends on $\Delta$.

The leading contribution of a fermion field was analyzed for spatial spheres in [25]. The case of spheres of arbitrary orientations in spacetime was computed in [7]. Calling $n_{A}$, $n_{B}$, to the future directed time-like unit vectors normal to the planes of the spheres, and $l$ to the unit spatial vector in the direction joining the centers of the two spheres, we have for a spinor primary field

$$
I_{\text {ferm }}(A, B)=2^{\left[\frac{d}{2}\right]+1} c(\Delta)\left[2\left(n_{A} \cdot l\right)\left(n_{B} \cdot l\right)-\left(n_{A} \cdot n_{B}\right)\right]\left(\frac{R_{A} R_{B}}{L^{2}}\right)^{2 \Delta}+\ldots
$$

Interestingly, an explicit calculation for the EMI model shows that the same tensorial structure appears,

$$
I_{\mathrm{EMI}}(A, B)=\frac{4(d-1)(d-2) \pi^{d-1} \kappa_{(d)}}{\Gamma\left(\frac{d+1}{2}\right)^{2}}\left[2\left(n_{A} \cdot l\right)\left(n_{B} \cdot l\right)-\left(n_{A} \cdot n_{B}\right)\right]\left(\frac{R_{A} R_{B}}{L^{2}}\right)^{d-1}+\ldots
$$

This follows from doing the integrals in (1.12) and expanding for long-distance. We will be more explicit about this computation in section 3 below, where we compute the full form of the EMI mutual information for boosted spheres at any distance.

Now, the comparison of the EMI expression with eq. (2.1) shows that the lowest dimensional primary of the EMI model must have dimension $\Delta=(d-1) / 2$. Because of the unitarity bounds $[26,27]$, this can be only the case of either a scalar field or a fermion field. Comparing with equations (2.2) and (2.3) we see that the tensor structure of the contribution is only compatible with a fermion field. This fermion field saturates the unitarity bound and is a free field of helicity $1 / 2$. We cannot have a contribution of a scalar field of the same dimension as it would spoil the tensor structure in eq. (2.4).

In order to compare the free fermion with the EMI, it is useful to calibrate the coefficient $\kappa_{(d)}$ to exactly match the long-distance free fermion contribution by taking

$$
\kappa_{(d)}=\frac{2^{\left[\frac{d}{2}\right]} \Gamma(d-2) \Gamma\left(\frac{d+1}{2}\right)^{2}}{8 \pi^{d-3 / 2} \Gamma\left(d+\frac{1}{2}\right)},
$$

although note that, in principle, the number of free fermionic fields in the EMI could be any integer. 


\subsection{Two dimensions}

As it turns out, in $d=2$ the EMI model indeed coincides with the free fermion. For the latter, in the case of two arbitrary regions formed by intervals, whose projections on the null axis $x^{ \pm}=x^{1} \pm x^{0}$ are the multi-interval sets $A^{ \pm}$and $B^{ \pm}$, we have $[22,28]$

$$
I_{\text {ferm }}(A, B)=\frac{1}{6}\left[\int_{A^{+}} \mathrm{d} x^{+} \int_{B^{+}} \mathrm{d} y^{+} \frac{1}{\left(x^{+}-y^{+}\right)^{2}}+\int_{A^{-}} \mathrm{d} x^{-} \int_{B^{-}} \mathrm{d} y^{-} \frac{1}{\left(x^{-}-y^{-}\right)^{2}}\right] .
$$

This expression is bilinear in the two regions and gives an extensive MI, $I_{3} \equiv 0$. It coincides with eq. (1.11) for $\kappa_{(2)}=1 / 6$.

The reason for extensivity in this case can be tracked to bosonization in $d=2$ [22]. The Rényi operators in the replicated theory are products of exponentials of the current for $n$ different decoupled free fermion fields. The free fermion current can be written as a linear expression in a dual free scalar field. Therefore the correlators of the Rényi twist operators are correlators of exponentials of free fields. These are exponentials of bilinear expressions, and upon taking the logarithm, the Rényi entropies turn out to be bilinear in the two regions. The entropy inherits this same form.

\subsection{Not a free fermion for $d>2$}

In dimensions higher than two, the free fermion does not have extensive mutual information. This can be seen in several ways by comparing the EMI results for various entangling regions with the analogous free fermion ones. In this subsection, we perform several comparisons of that kind and observe that the discrepancy in various charges characterizing the free fermion and the EMI tends to grow as the spacetime dimension increases. For many of the comparisons, we normalize the EMI results so that the long-distance coefficient matches the free fermion one, as in eq. (2.5). We also try with other ratios.

Three dimensions. Let us start with the $d=2+1$ case. Consider first the mutual information for two regions with parallel boundaries of size $L$ separated a short distance $r \ll L$. As reviewed in appendix A, this behaves, for any CFT as

$$
I=\frac{k^{(3)} L}{r}+\ldots
$$

where $k^{(3)}$ is a theory-dependent coefficient which matches the universal coefficient in the entanglement entropy corresponding to a long and thin strip of dimensions $L \times r$. For the EMI model, the result for the general-dimension version of $k^{(d)}$ appears in eq. (A.5). Particularizing to $d=3$, we have

$$
k_{\mathrm{EMI}}^{(3)}=2 \pi \kappa_{(3)} \stackrel{(2.5)}{=} \frac{4}{15 \pi} \simeq 0.0849,
$$

where in the second equality we fixed the value of $\kappa_{(3)}$ calibrated so that the long-distance coefficient for a pair of disks matches the free fermion one - see eq. (2.5) above. For a Dirac field, the analogous coefficient reads [29]

$$
k_{\text {ferm }}^{(3)} \simeq 0.0722 .
$$

Hence, we observe that both values are clearly different, the discrepancy being $\sim 15 \%$. 
On the other hand, if one considers the mutual information for two concentric disks of radii $R_{A}<R_{B}$, the EMI result obtained in the following section for $d=3$ becomes

$$
I_{\mathrm{EMI}}=8 \pi^{2} \kappa_{(3)} \frac{\left(R_{A} / R_{B}\right)^{2}}{\left[1-\left(R_{A} / R_{B}\right)^{2}\right]} .
$$

As explained in the appendix, when the disks are very close to each other, $R_{A}=R-\delta / 2$, $R_{B}=R+\delta / 2, \delta \ll R$, we can expand this expression to get

$$
I_{\mathrm{EMI}}=k_{\mathrm{EMI}}^{(3)} \frac{2 \pi R}{\delta}-2 F_{\mathrm{EMI}}^{(3)}, \quad \text { where } \quad F_{\mathrm{EMI}}^{(3)}=2 \pi^{2} \kappa_{(3)} \stackrel{(2.5)}{=} \frac{4}{15} \simeq 0.2667,
$$

is the $F$-term appearing in the entanglement entropy of a disk region, and the strip coefficient $k_{\mathrm{EMI}}^{(3)}$ appears in the area-law piece. In the case of the Dirac fermion, $F^{(3)}$ is known analytically and reads $[30,31]$

$$
F_{\text {ferm }}^{(3)}=\frac{1}{8}\left(2 \log 2+\frac{3}{\pi^{2}} \zeta(3)\right) \simeq 0.21896 .
$$

Hence, we again observe a considerable difference between models, in this case around $\sim 18 \%$.

Observe that by using the value of $\kappa_{(3)}$ calibrated with the long distance coefficient for different charges such as $k^{(3)}$ or $F^{(3)}$, what we are effectively doing is comparing the quotient of such quantities divided by the long distance coefficient in both models. We can naturally perform additional comparisons by dividing by other charges. For instance, comparing the quotients $F^{(3)} / k^{(3)}$ instead, one obtains

$$
\frac{F_{\mathrm{EMI}}^{(3)}}{k_{\mathrm{EMI}}^{(3)}}=\pi, \quad \frac{F_{\text {ferm }}^{(3)}}{k_{\text {ferm }}^{(3)}} \simeq 3.033,
$$

which are much closer, only differing by $\sim 3.5 \%$.

Additional comparisons can be made by considering an entangling region with a corner of angle $\theta$. In that case, the entanglement entropy contains a logarithmic divergence of the form $[32,33]$

$$
\left.S_{\mathrm{EE}}\right|_{\log }=-a(\theta) \log (R / \delta),
$$

where $a(\theta)$ is a universal function of the corner opening angle. In the case of the EMI, this function turns out to be given by $[16,34]$

$$
a_{\mathrm{EMI}}(\theta)=2 \kappa_{(3)}[1+(\pi-\theta) \cot \theta] .
$$

For very sharp and almost-smooth corners, $a(\theta)$ behaves, on general grounds, as [32]

$$
a(\theta \rightarrow 0)=\frac{k^{(3)}}{\theta}+\ldots, \quad a(\theta \rightarrow \pi)=\sigma(\pi-\theta)^{2}+\ldots
$$

In this expression, $k^{(3)}$ is the strip coefficient appearing in eq. (2.7), and $\sigma$ turns out to be related to the stress-tensor two-point function charge $C_{T}{ }^{3}$ as $[18,36]$

$$
\sigma=\frac{\pi^{2}}{24} C_{T} .
$$

\footnotetext{
${ }^{3}$ For a general CFT in $d$ dimensions, the tensorial structure of the flat-space stress tensor two-point function is fully determined by conformal symmetry up to a theory-dependent constant: $\left\langle T_{a b}(x) T_{c d}(0)\right\rangle_{\mathbb{R}^{d}}=$ $C_{T} \mathcal{I}_{a b, c d} /|x|^{2 d}$, where $\mathcal{I}_{a b, c d}$ is a fixed dimensionless expression [35].
} 
Using eq. (2.15), we can then obtain the would-be value of $C_{T}^{\mathrm{EMI}}$ in case this represented an actual QFT. The result, and the analogous one for a free fermion [35] are given respectively by

$$
C_{T}^{\mathrm{EMI}}=\frac{16 \kappa_{(3)}}{\pi^{2}} \stackrel{\stackrel{(2.5)}{=}}{=} \frac{32}{15 \pi^{2}} \simeq 0.0219, \quad C_{T}^{\mathrm{ferm}}=\frac{3}{16 \pi^{2}} \simeq 0.019 .
$$

These two differ by $\sim 13 \%$. We can also compare the results with $F^{(3)}$ or $k^{(3)}$. For instance, we have

$$
\frac{F_{\mathrm{EMI}}^{(3)}}{C_{T}^{\mathrm{EMI}}}=\frac{\pi^{4}}{8} \simeq 12.1761, \quad \frac{F_{\text {ferm }}^{(3)}}{C_{T}^{\text {ferm }}}=\frac{\pi^{2}}{3}\left(2 \log 2+\frac{3 \zeta(3)}{\pi^{2}}\right) \simeq 11.5256,
$$

which differ by $\sim 5.3 \%$. We observe that comparing the various charges with the longdistance coefficient tends to make the agreement with the fermion worse than when comparing the other charges amongst each other.

Four dimensions. In this case we can use Solodukhin's formula for the entanglement entropy universal term to evaluate the trace-anomaly coefficients $a$ and $c$ [37]. In particular for entangling surfaces corresponding to spheres and cylinders the relevant terms read, respectively,

$$
\left.S_{\mathrm{EE}}\right|_{\mathrm{sphere}}=-4 a \log (R / \delta),\left.\quad S_{\mathrm{EE}}\right|_{\mathrm{cylinder}}=-\frac{c}{2} \frac{L}{R} \log (R / \delta) .
$$

For the EMI, simple calculations yield the right expressions appearing in eq. (2.20), where

$$
a_{\mathrm{EMI}}=\pi^{2} \kappa_{(4)} \stackrel{(2.5)}{=} \frac{3}{70} \simeq 0.0429, \quad c_{\mathrm{EMI}}=\frac{3 \pi^{2} \kappa_{(4)}}{2} \stackrel{\stackrel{(2.5)}{=}}{=} \frac{9}{140} \simeq 0.0643, \quad \text { so } \quad \frac{a_{\mathrm{EMI}}}{c_{\mathrm{EMI}}}=\frac{2}{3} .
$$

It is an interesting fact that the would-be CFT represented by the EMI lies within the range of values allowed by the unitarity bounds [26, 27]

$$
\frac{1}{3} \leq \frac{a}{c} \leq \frac{31}{18}
$$

Naturally, the free fermion also satisfies the bounds, but one finds in that case $[26,38,39]$

$$
a_{\text {ferm }}=\frac{11}{360} \simeq 0.0306 \quad c_{\text {ferm }}=\frac{1}{20}=0.05 \quad \frac{a_{\text {ferm }}}{c_{\text {ferm }}}=\frac{11}{18} \simeq 0.6111 .
$$

The discrepancies range from $\sim 8.33 \%$ for the ratios $a / c$ to $\sim 29 \%$ for the $a$ 's normalized by the long-distance coefficient.

We can make another comparison using the values of the coefficient characterizing the mutual information of two parallel regions which are very close to each other - see appendix A. The result for $k^{(4)}$ for a Dirac fermion is given by [29]

$$
k_{\text {ferm }}^{(4)} \simeq 0.0215 .
$$

whereas for the EMI we find from eq. (A.5)

$$
k_{\mathrm{EMI}}^{(4)}=2 \pi \kappa_{(4)} \stackrel{(2.5)}{=} \frac{3}{35 \pi} \simeq 0.0273,
$$


which differ by $\sim 21 \%$. On the other hand, the ratios $a / k^{(4)}$ are a bit closer, namely

$$
\frac{a_{\mathrm{EMI}}}{k_{\mathrm{EMI}}^{(4)}}=\frac{\pi}{2} \simeq 1.5708, \quad \frac{a_{\mathrm{ferm}}}{k_{\mathrm{ferm}}^{(4)}} \simeq 1.4199,
$$

which is $\sim 9.6 \%$ off.

Five dimensions. Moving to $d=4+1$, we can consider for instance the strip coefficient $k^{(5)}$ and the one appearing in the entanglement entropy across a $\mathbb{S}^{3}$, which we denote $F^{(5)}$. As explained in appendix A, both can be obtained from the mutual information of a pair of concentric spheres of radii $R_{A}=R-\delta / 2, R_{B}=R+\delta / 2, \delta \ll R$, analogously to lower dimensions. In this case we have, on general grounds

$$
I=k^{(5)} \frac{2 \pi^{2} R^{3}}{\delta^{3}}+\cdots+2 F^{(5)},
$$

where the dots denote subleading divergences. For the EMI, we find

$$
k_{\mathrm{EMI}}^{(5)}=\frac{\pi^{2} \kappa_{(5)}}{2} \stackrel{(2.5)}{=} \frac{64}{945 \pi^{2}} \simeq 0.00686, \quad F_{\mathrm{EMI}}^{(5)}=\pi^{4} \kappa_{(5)} \stackrel{(2.5)}{=} \frac{128}{945} \simeq 0.135,
$$

SO

$$
\frac{F_{\mathrm{EMI}}^{(5)}}{k_{\mathrm{EMI}}^{(5)}}=2 \pi^{2} \simeq 19.7392
$$

On the other hand, for the fermion, we have [29, 30]

$$
k_{\text {ferm }}^{(5)}=0.0052, \quad F_{\text {ferm }}^{(5)}=\frac{1}{64}\left[6 \log 2+\frac{10 \zeta(3)}{\pi^{2}}+\frac{15 \zeta(5)}{\pi^{4}}\right] \simeq 0.0865, \quad \text { so } \quad \frac{F_{\text {ferm }}^{(5)}}{k_{\text {ferm }}^{(5)}} \simeq 16.636 .
$$

Hence, in this case the differences range from $\sim 15.7 \%$ for the quotient $F_{\text {ferm }}^{(5)} / k_{\text {ferm }}^{(5)}$ to $\sim 35.9 \%$ for the $F_{\text {ferm }}^{(5)}$ normalized by the long-distance coefficient.

Six dimensions. In $d=5+1$ we can consider again the coefficient $A^{(6)}$ which appears weighting the Euler density in the trace anomaly - see eq. (A.7) below. This we can compare with the strip one, $k^{(6)}$. From eq. (A.5) and eq. (A.8) we have for the EMI model

$$
k_{\mathrm{EMI}}^{(6)}=\frac{\pi^{2}}{3} \kappa_{(6)} \stackrel{(2.5)}{=} \frac{10}{231 \pi^{2}} \simeq 0.00439, \quad A_{\mathrm{EMI}}^{(6)}=\frac{\pi^{4}}{6} \kappa_{(6)} \stackrel{(2.5)}{=} \frac{5}{231} \simeq 0.0216,
$$

so

$$
\frac{A_{\mathrm{EMI}}^{(6)}}{k_{\mathrm{EMI}}^{(6)}}=\frac{\pi^{2}}{2} \simeq 4.9348
$$

On the other hand, the analogous coefficients for the free fermion can be extracted from refs. [29] and [40, 41], respectively, and read

$$
k_{\text {ferm }}^{(6)} \simeq 0.00325, \quad A_{\text {ferm }}^{(6)}=\frac{191}{15120} \simeq 0.0126 \quad \text { so } \quad \frac{A_{\text {ferm }}^{(6)}}{k_{\text {ferm }}^{(6)}} \simeq 3.889 .
$$

In this case, discrepancies vary from $\sim 26 \%$ for the $k^{(6)}$ 's normalized by the long distance coefficient to $\sim 41.7 \%$ for the $A^{(6)}$ 's normalized by the same. 
Seven dimensions. As a final case, we consider $d=6+1$. Once more, we compare $k^{(7)}$ and $F^{(7)}$. Using the formulas in the appendix, we have for the EMI

$$
k_{\mathrm{EMI}}^{(7)}=\frac{\pi^{3}}{16} \kappa_{(7)} \stackrel{(2.5)}{=} \frac{256}{5005 \pi^{3}} \simeq 0.00165, \quad F_{\mathrm{EMI}}^{(7)}=\frac{\pi^{6}}{12} \kappa_{(7)} \stackrel{(2.5)}{=} \frac{1024}{15015} \simeq 0.0681 .
$$

so

$$
\frac{F_{\mathrm{EMI}}^{(7)}}{k_{\mathrm{EMI}}^{(7)}}=\frac{4 \pi^{3}}{3} \simeq 41.342 .
$$

On the other hand, for a free fermion we have [29, 30]

$$
k_{\text {ferm }}^{(7)} \simeq 0.0012, \quad F_{\text {ferm }}^{(7)}=\frac{1}{512}\left[20 \log 2+\frac{518 \zeta(3)}{15 \pi^{2}}+\frac{70 \zeta(5)}{\pi^{4}}+\frac{63 \zeta(7)}{\pi^{6}}\right] \simeq 0.0369 .
$$

so

$$
\frac{F_{\text {ferm }}^{(7)}}{k_{\text {ferm }}^{(7)}} \simeq 30.73
$$

The greatest difference $(\sim 45.8 \%)$ appears in the $F^{(7)}$ 's normalized by the long distance coefficient.

All in all, we observe that both models are clearly different for $d>2$ and they seem to increasingly deviate from each other as the number of dimensions grows.

\section{Current conformal block equals EMI for spherical regions}

In this section we compute the MI for two spatially separated and arbitrarily boosted spheres for the EMI model. Remarkably, the result turns out to be identical to the one corresponding to the conformal block $G_{d-1,1}^{d}$ associated to the conserved current operator made out of two free fermions.

In Minkowski space, the configuration space of "boosted" spherical regions is determined by the tips of their causal developments. Thus, for two spheres, the mutual information is a function of the four associated spacetime points. Furthermore, conformal symmetry implies that such dependence can only come through two independent conformally invariant cross ratios, say, $u$ and $v$ - see below for the precise definitions.

In the long-distance regime, an OPE analysis in the replicated theory suggests that such function should be given in terms of the conformal blocks associated to each primary operator of the replica theory. The following expansion is implied [42-44],

$$
I(A, B)=\sum_{\Delta, J} b_{\Delta, J} G_{\Delta, J}^{d}(u, v) .
$$

Here, $\Delta$ and $J$ are the scaling dimension and spin of the primary modules in the replica theory, ${ }^{4}$ respectively, and the coefficients $b_{\Delta, J}$ of each contributing conformal block can be obtained via an analysis along the lines of $[3,4]$ - this was recently reviewed, generalized and expressed in a compact form in [7]. In particular, for spheres lying on the same time

\footnotetext{
${ }^{4}$ Notice that it might be the case that the operator $\mathcal{O}_{\Delta}$ is a primary operator in the replica theory but it is not a primary in the original CFT.
} 
slice, the sum of the coefficients associated to the leading contribution of a primary operator in the original CFT takes the simple form

$$
\sum_{J} b_{\Delta, J}=\frac{\sqrt{\pi} \Gamma(\Delta+1) \operatorname{dim}(\mathcal{R})}{4^{\Delta+1} \Gamma\left(\Delta+\frac{3}{2}\right)},
$$

where $\mathcal{R}$ is the Lorentz representation of the CFT primary operator that contributes to $I(A, B)$. For other primary operators in the replica theory, one can either use the analysis of [7] adapted to such computation or the explicit formulas from [42-44].

Now, if we are able to compute the mutual information for boosted spherical regions in the EMI model, equating such result to the r.h.s. of eq. (3.1) would allow us to extract the full set of $\{\Delta, J\}$ of the primary modules contributing to the mutual information of the putative CFT corresponding to this model. From the analysis of section 2 we know that the EMI is dominated at long distances by the contribution coming from a free fermion. Comparing that result with eq. (3.1), we find that the leading replica operator that contributes to $I_{\mathrm{EMI}}(A, B)$ - through analytic extension from the contribution to the Renyi entropies to replica number $n=1$ - is precisely a conserved current operator made out of two free fermions in two replicas $i, j, i \neq j$,

$$
J_{\mu}=\bar{\psi}_{i} \gamma_{\mu} \psi_{j},
$$

with $\Delta=d-1$ and $J=1$. Taking this as our starting point, we would like to find the set of operators that contribute to $I_{\mathrm{EMI}}(A, B)$ via a recursive process in which we consecutively subtract the various conformal-block contributions to the full expression of the EMI.

In more detail, the idea is the following. We start assuming that $I_{\mathrm{EMI}}(A, B)$ has an expansion as the one given in eq. (3.1), thus

$$
I_{\mathrm{EMI}}(A, B)=\sum_{\Delta, J} b_{\Delta, J} G_{\Delta, J}^{d}(u, v)
$$

for a set $\{\Delta, J\}$ to be determined. Since at long distances the leading contribution is consistent with the conformal block of an operator with $\Delta=d-1$ and $J=1$, and such operator can only be associated to the leading contribution of a free fermion, we subtract such contribution to $I_{\mathrm{EMI}}(A, B)$ and repeat the analysis for the remaining expression. This is, after the first iteration we have

$$
I_{\mathrm{EMI}}^{(1)}(A, B) \equiv I_{\mathrm{EMI}}(A, B)-b_{d-1,1}^{\text {(ferm) }} G_{d-1,1}^{d}(u, v)
$$

with [44]

$$
b_{d-1,1}^{(\mathrm{ferm})}=2^{\left[\frac{d}{2}\right]+1} \frac{\sqrt{\pi} \Gamma(d)}{4^{d} \Gamma\left(d+\frac{1}{2}\right)},
$$

which has the expansion

$$
I_{\mathrm{EMI}}^{(1)}(A, B)=\sum_{\{\Delta, J\} \neq\{d-1,1\}} b_{\Delta, J} G_{\Delta, J}^{d}(u, v) .
$$




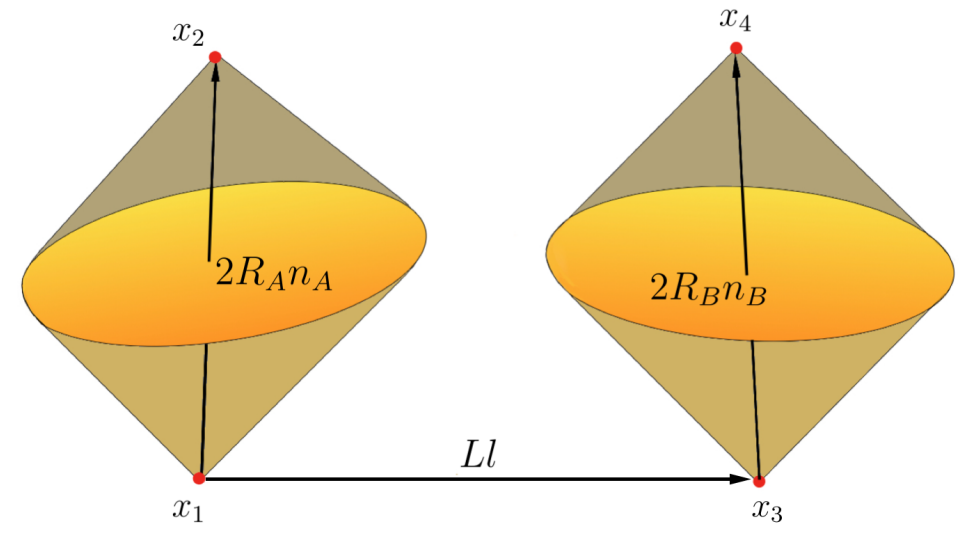

Figure 1. Schematic representation of the geometric setup. We consider two boosted spheres $\mathbb{S}_{A}^{d-2}$, $\mathbb{S}_{B}^{d-2}$, characterized by their causal tips $x_{1}, x_{2}$ and $x_{3}, x_{4}$, respectively. The vector joining $x_{1}$ and $x_{3}$ is denoted $L l$ and the ones connecting the diamond tips of each sphere by $2 R_{A} n_{A}$ and $2 R_{B} n_{B}$, respectively.

Studying the long-distance behavior of (3.5) allows us to deduce the associated values of $\{\Delta, J\}$ for the next-to-leading contributing operator and by reading the associated coefficient we could deduce to which operator that corresponds to. We repeat the process until we have exhausted all the operators that contribute to the l.h.s. of (3.4) or until we have zero remanent. The process will turn out to be surprisingly short, as we will find $I_{\mathrm{EMI}}^{(1)}(A, B)=0$.

\subsection{EMI for boosted spherical regions}

The first step in our program is to compute the EMI for relatively boosted spherical regions. In such case the EMI formula is given by eq. (1.12) or, equivalently, by eq. (1.12). Thus for the case of relatively boosted spheres the mutual information can be written as

$$
I_{\mathrm{EMI}}(A, B)=2 \kappa_{(d)} \int_{\mathbb{S}_{A}^{d-2}} \mathrm{~d} \sigma_{A} \int_{\mathbb{S}_{B}^{d-2}} \mathrm{~d} \sigma_{B} \frac{\left(n_{A} \cdot n_{B}\right)\left(\bar{n}_{A} \cdot \bar{n}_{B}\right)-\left(n_{A} \cdot \bar{n}_{B}\right)\left(\bar{n}_{A} \cdot n_{B}\right)}{\left|\mathbf{r}_{A}-\mathbf{r}_{B}\right|^{2(d-2)}},
$$

where we modified the notation slightly with respect to eq. (1.12) for future convenience. We describe our geometric setup through the tips of the causal diamonds whose past and future null cones intersect at the corresponding spheres. We use $x_{1}$ and $x_{2}$ to label the past and future tips of the sphere $A$, respectively, and analogously with $x_{3}$ and $x_{4}$ for the sphere $B$. In figure 1 , we give a schematic representation of this setup.

Given these four spacetime points, the mutual information will be a function of the usual conformal invariant cross ratios $u, v$, which are given by

$$
u \equiv \frac{\left|x_{1}-x_{2}\right|^{2}\left|x_{3}-x_{4}\right|^{2}}{\left|x_{1}-x_{3}\right|^{2}\left|x_{2}-x_{4}\right|^{2}}, \quad \text { and } \quad v \equiv \frac{\left|x_{1}-x_{4}\right|^{2}\left|x_{2}-x_{3}\right|^{2}}{\left|x_{1}-x_{3}\right|^{2}\left|x_{2}-x_{4}\right|^{2}} .
$$

However, for our convenience we will work instead with the conformally independent parameters

$$
\chi_{1}=\frac{\left|x_{1}-x_{2}\right|\left|x_{3}-x_{4}\right|}{\left|x_{1}-x_{3}\right|\left|x_{2}-x_{4}\right|} \quad \text { and } \quad \chi_{2}=\frac{\left|x_{1}-x_{2}\right|\left|x_{3}-x_{4}\right|}{\left|x_{1}-x_{4}\right|\left|x_{2}-x_{3}\right|}
$$


which are related to $u$ and $v$ via

$$
u=\chi_{1}^{2}, \quad \text { and } \quad v=\frac{\chi_{1}^{2}}{\chi_{2}^{2}} .
$$

For the above geometric set up we can write $x_{2}-x_{1}=2 R_{A} n_{A}, x_{4}-x_{3}=2 R_{B} n_{B}$, where $R_{A}$ and $R_{B}$ are the radii of the spheres $A$ and $B$ respectively, and $n_{A}$ and $n_{B}$ are the future directed unit time-like vectors pointing from the past to the future cone tips of each sphere. We write the difference $x_{3}-x_{1}=L l$, where $L$ is the distance between the past tip cones of the spheres and $l$ is a unit space-like vector.

With the above identifications the conformal parameters become

$$
\chi_{1}=\frac{4 R_{A} R_{B}}{L\left|2 R_{A} n_{A}-L l-2 R_{B} n_{B}\right|}, \quad \text { and } \quad \chi_{2}=\frac{4 R_{A} R_{B}}{\left|2 R_{A} n_{A}-L l\right|\left|2 R_{B} n_{B}+L l\right|},
$$

with ranges $\chi_{1} \in(0,1), \chi_{2} \in(0, \infty)$ with $\chi_{2}>\chi_{1}$ for spatially separated spheres.

Although we are interested in the above geometric set up, we can simplify our computation, without loss of generality, by performing a conformal transformation which makes the centers of the spheres coincide. In that case, the conformal parameters take the values

$$
\chi_{1}=\frac{4 R_{A} R_{B}}{\left|R_{A} n_{A}-R_{B} n_{B}\right|^{2}}, \quad \text { and } \quad \chi_{2}=\frac{4 R_{A} R_{B}}{\left|R_{A} n_{A}+R_{B} n_{B}\right|^{2}},
$$

where we use the relation $x_{3}-x_{1}=R_{A} n_{A}-R_{B} n_{B}=L l$ that follows from the equal-center condition. We parametrize the inner product between $n_{A}$ and $n_{B}$ by a boost parameter $\beta$ via

$$
n_{A} \cdot n_{B} \equiv-\cosh \beta,
$$

and introduce the parameter $0 \leq x \leq 1$, defined as

$$
x \equiv \frac{2 R_{A} R_{B}}{R_{A}^{2}+R_{B}^{2}} .
$$

The parameters $\chi_{1}$ and $\chi_{2}$ can then be written in terms of $x$ and $\beta$ as

$$
\chi_{1}=\frac{2 x}{|1-x \cosh \beta|}, \quad \text { and } \quad \chi_{2}=\frac{2 x}{(1+x \cosh \beta)} .
$$

In this case, $\chi_{1}>\chi_{2}$ and $\chi_{2} \in(0,1), \chi_{1} \in(0, \infty)$ so essentially the conformal transformation interchanged the rôles of $\chi_{1}$ and $\chi_{2}$, which is a natural thing for an inversion like the one we are considering here. Given $R_{A}$ and $R_{B}$, there is a critical value of $\beta$ for which $\chi_{1}$ is ill-defined. From eq. (3.13) one can see that this happens when the tip cones are null-like separated, $R_{A} n_{A}-R_{B} n_{B}=$ null vector, or in other words, when the causal cones have a common boundary. Assuming the casual cones obey a strict inclusion relation, then

$$
0 \leq \cosh \beta<\frac{R_{A}^{2}+R_{B}^{2}}{2 R_{A} R_{B}} .
$$

This in turn is equivalent to say that the cone tips are time-like separated. To describe such configurations, the absolute value in (3.16) is thus unnecessary. 
We will write down a formula for the EMI in the above set up as a funciton of $x$ and $\beta$. Then, whenever necessary, we will go back and rewrite it in terms of the perhaps more familiar $u$ and $v$ variables. Points on the spheres $A$ and $B$ will be denoted by $\mathbf{r}_{A}$ and $\mathbf{r}_{B}$ respectively. $n_{A}$ and $n_{B}$ are also the future oriented normal vectors to the spheres $A$ and $B$ respectively. Thus the points on the spheres satisfy

$$
\mathbf{r}_{A}^{2}=R_{A}^{2}, \quad \mathbf{r}_{A}^{2}=R_{A}^{2}, \quad \text { and } \quad n_{A} \cdot \mathbf{r}_{A}=0, \quad n_{B} \cdot \mathbf{r}_{B}=0 .
$$

Since the spheres are concentric, we can write $\mathbf{r}_{A}=R_{A} \bar{n}_{A}$ and $\mathbf{r}_{B}=R_{B} \bar{n}_{B}$ where $\bar{n}_{A}, \bar{n}_{B}$ are the unit vectors normal to each sphere on their respective hyperplanes.

We can choose a coordinate system such that the sphere $A$ lies on the $t=$ constant slice while the sphere $B$ is boosted along the $\hat{z}$ direction with the respect to $A$. We have then

$$
n_{A}=\hat{t}, \quad \text { and } \quad n_{B}=\cosh \beta \hat{t}+\sinh \beta \hat{z} .
$$

Given eq. (3.19), we can find the solutions to eq. (3.18) as

$$
\begin{aligned}
& \mathbf{r}_{A}=R_{A} \bar{n}_{A}=R_{A}\left(\left|\vec{\Omega}_{A}\right| \hat{\Omega}_{A}+\Omega_{A, z} \hat{z}\right), \\
& \mathbf{r}_{B}=R_{B} \bar{n}_{B}=R_{B}\left(\sinh \beta \Omega_{B, z} \hat{t}+\left|\vec{\Omega}_{B}\right| \hat{\Omega}_{B}+\cosh \beta \Omega_{B, z} \hat{z}\right) .
\end{aligned}
$$

The relative boost partially breaks the spherical symmetry and thus we have separated the angular variables describing a unit sphere by $|\vec{\Omega}| \hat{\Omega}+\Omega_{z} \hat{z}$ above. We will integrate first the sphere $A$ which is the most symmetric one in the above coordinates. To simplify our computation even further, we rotate the coordinate system such that the new $\hat{z}$ coincides with the radial direction of the sphere $B$. This means that the vectors transform as

$$
\begin{aligned}
& \mathbf{r}_{B}=R_{B}\left(\sinh \beta \cos \theta_{B} \hat{t}+\sqrt{1+\sinh ^{2} \beta \cos ^{2} \theta_{B}} \hat{z}\right), \\
& n_{B}=\left(\cosh \beta \hat{t}-\sinh \beta \sin \phi_{B} \hat{\Omega}_{B}+\sinh \beta \cos \phi_{B} \hat{z}\right),
\end{aligned}
$$

where we used $\Omega_{B, z}=\cos \theta_{B}$, and the rotation angle $\phi_{B}$ can be seen, from the orthogonality condition (3.18), to be given by

$$
\cos \phi_{B}=\frac{\cosh \beta \cos \theta_{B}}{\sqrt{1+\sinh ^{2} \beta \cos ^{2} \theta_{B}}} \quad \text { and } \quad \sin \phi_{B}=\frac{\sin \theta_{B}}{\sqrt{1+\sinh ^{2} \beta \cos ^{2} \theta_{B}}} .
$$

Since the sphere $A$ is spherically symmetric, we can use expressions (3.20) and (3.18) for $\mathbf{r}_{A}$ and $n_{A}$. We are ready now to write down the integral expressing the EMI for two spheres using (3.8). The main ingredients are

$$
\begin{aligned}
& \left|\mathbf{r}_{A}-\mathbf{r}_{B}\right|^{2}=\left|R_{A}^{2}+R_{B}^{2}-2 R_{A} R_{B} \sqrt{1+\sinh ^{2} \beta \cos ^{2} \theta_{B}} \cos \theta_{A}\right|, \\
& \left(n_{A} \cdot n_{B}\right)\left(\bar{n}_{A} \cdot \bar{n}_{B}\right)-\left(n_{A} \cdot \bar{n}_{B}\right)\left(\bar{n}_{A} \cdot n_{B}\right)=-\frac{\cosh \beta \cos \theta_{A}}{\sqrt{1+\sinh ^{2} \beta \cos ^{2} \theta_{B}}},
\end{aligned}
$$

where in equation (3.25) we have ignored terms proportional to $\hat{\Omega}_{B} \cdot \hat{\Omega}_{A}$ since those integrate to zero. Likewise, we can explicitly integrate the angles $\hat{\Omega}_{B}$ and $\hat{\Omega}_{A}$ for each sphere 
which entails the replacement

$$
\int_{\mathbb{S}_{A}^{d-2}} \mathrm{~d} \sigma_{A} \int_{\mathbb{S}_{B}^{d-2}} \mathrm{~d} \sigma_{B} \rightarrow \frac{4 R_{A}^{d-2} R_{B}^{d-2} \pi^{d-2}}{\Gamma\left(\frac{d-2}{2}\right)^{2}} \int_{0}^{\pi} \mathrm{d} \theta_{A} \sin ^{d-3} \theta_{A} \int_{0}^{\pi} \mathrm{d} \theta_{B} \sin ^{d-3} \theta_{B} .
$$

Putting everything together, we finally have

$$
\begin{aligned}
I_{\mathrm{EMI}}= & \frac{8 \kappa_{(d)} R_{A}^{d-2} R_{B}^{d-2} \pi^{d-2}}{\Gamma\left(\frac{d-2}{2}\right)^{2}} \int_{0}^{\pi} \int_{0}^{\pi} \frac{\mathrm{d} \theta_{B} \mathrm{~d} \theta_{A} \sin ^{d-3} \theta_{B} \sin ^{d-3} \theta_{A}}{\left|R_{A}^{2}+R_{B}^{2}-2 R_{A} R_{B} \sqrt{1+\sinh ^{2} \beta \cos ^{2} \theta_{B}} \cos \theta_{A}\right|^{d-2}} \\
& \times \frac{\cosh \beta \cos \theta_{A}}{\sqrt{1+\sinh ^{2} \beta \cos ^{2} \theta_{B}}} .
\end{aligned}
$$

We can rewrite the above integral in terms of the parameter $x$ defined in eq. (3.15) and the function $f_{\beta}(\theta)$ defined by

$$
f_{\beta}(\theta) \equiv \sqrt{1+\sinh ^{2} \beta \cos ^{2} \theta} \quad \text { with } \quad x f_{\beta}(\theta)<1,
$$

which follows from eq. (3.17). Making these replacements, the EMI takes the form

$$
I_{\mathrm{EMI}}=\frac{8 \kappa_{(d)} 2^{2-d} x^{d-2} \pi^{d-2}}{\Gamma\left(\frac{d-2}{2}\right)^{2}} \cosh \beta \int_{0}^{\pi} \frac{\mathrm{d} \theta_{B} \sin ^{d-3} \theta_{B}}{f_{\beta}\left(\theta_{B}\right)} \int_{0}^{\pi} \frac{\mathrm{d} \theta_{A} \sin ^{d-3} \theta_{A} \cos \theta_{A}}{\left(1-x f_{\beta}\left(\theta_{B}\right) \cos \theta_{A}\right)^{d-2}} .
$$

The integral over $\theta_{A}$ can be carried out explicitly, and a convenient expression for it reads

$$
\begin{aligned}
\int_{0}^{\pi} \frac{\mathrm{d} \theta_{A} \sin ^{d-3} \theta_{A} \cos \theta_{A}}{\left(1-x f_{\beta}\left(\theta_{B}\right) \cos \theta_{A}\right)^{d-2}}= & \frac{\sqrt{\pi} \Gamma\left(\frac{d-2}{2}\right)}{\Gamma\left(\frac{d-1}{2}\right)}\left(\frac{d-2}{d-1}\right) \frac{x f_{\beta}\left(\theta_{B}\right)}{\left[1-x^{2} f_{\beta}^{2}\left(\theta_{B}\right)\right]^{d / 2}} \\
& \times{ }_{2} F_{1}\left[\frac{d}{2}, 1, \frac{d+1}{2} ;-\frac{x^{2} f_{\beta}^{2}\left(\theta_{B}\right)}{1-x^{2} f_{\beta}^{2}\left(\theta_{B}\right)}\right] .
\end{aligned}
$$

The final result is thus given as an integral over $\theta_{B}$, which we find convenient to express in terms of the variable $\xi \equiv \cos \theta_{B}$. Then, the final expression for the mutual information of two boosted spheres in the EMI model reads ${ }^{5}$

$$
I_{\mathrm{EMI}}=2 \gamma_{d} x^{d-1} \cosh \beta \int_{0}^{1} \mathrm{~d} \xi\left(1-\xi^{2}\right)^{\frac{d-4}{2}}{ }_{2} F_{1}\left[\frac{d}{2}, \frac{d-1}{2}, \frac{d+1}{2} ; x^{2}\left(1+\sinh ^{2} \beta \xi^{2}\right)\right],
$$

where

$$
\gamma_{d} \equiv \frac{2^{4} \kappa_{(d)} \pi^{d-\frac{3}{2}}}{2^{d-1} \Gamma\left(\frac{d-2}{2}\right) \Gamma\left(\frac{d-1}{2}\right)}\left(\frac{d-2}{d-1}\right) .
$$

The result is a function of the cross ratio defined in eq. (3.15) above and of the boost parameter $\beta$ introduced in eq. (3.14).

\footnotetext{
${ }^{5}$ To arrive at this expression we also use the identity

$$
{ }_{2} F_{1}(a, b, c ; z)=(1-z)^{-a}{ }_{2} F_{1}\left[a, c-b, c ; \frac{z}{z-1}\right] .
$$
}


For comparison purposes, it is important to write this back in terms of the $u$ and $v$ parameters. First from (3.16) one can solve for $x$ and $\cosh \beta$ as a function of $\chi_{1}$ and $\chi_{2}$,

$$
\frac{1}{x}=\frac{1}{\chi_{2}}+\frac{1}{\chi_{1}} \text { and } \quad \cosh \beta=\frac{1}{\chi_{2}}-\frac{1}{\chi_{1}} .
$$

Using (3.11) we can rewrite these in terms of $u$ and $v$ as

$$
x=\frac{\sqrt{u}}{1+\sqrt{v}}, \quad \cosh \beta=\frac{\sqrt{v}-1}{\sqrt{u}} .
$$

Notice that the trivial inequality $\cosh \beta>1$ implies $\sqrt{v} \geq 1+\sqrt{u}$, and the constraints $0 \leq x \leq 1$ and $0 \leq x \cosh \beta \leq 1$ are trivial consequences of this relation. In terms of $u$ and $v$ the final expression for the EMI reads

$$
\begin{aligned}
I_{\mathrm{EMI}}= & -2 \gamma_{d}\left(\frac{\sqrt{u}}{1+\sqrt{v}}\right)^{d-2}\left(\frac{1-\sqrt{v}}{1+\sqrt{v}}\right) \\
& \times \int_{0}^{1} \mathrm{~d} \xi\left(1-\xi^{2}\right)^{\frac{d-4}{2}}{ }_{2} F_{1}\left[\frac{d}{2}, \frac{d-1}{2}, \frac{d+1}{2} ; \frac{(1-\sqrt{v})^{2} \xi^{2}+u\left(1-\xi^{2}\right)}{(1+\sqrt{v})^{2}}\right] .
\end{aligned}
$$

Before diving into a detailed analysis of this result, let us study certain limiting cases.

\subsubsection{Long distance}

Let us consider the long-distance behavior of eq. (3.32) — or equivalently, eq. (3.36). To this end we need to go back to the disjoint-spheres geometry. In particular, the relation between the parameters $x$ and $\cosh \beta$, and $\chi_{1}$ and $\chi_{2}$, which was derived assuming the causal cones of the spheres to be nested, needs to be modified. The modification entails a simple interchange between $\chi_{1}$ and $\chi_{2}$. Indeed, for disjoint spheres, (3.34) becomes

$$
\frac{1}{x}=\frac{1}{\chi_{2}}+\frac{1}{\chi_{1}} \text { and } \quad \cosh \beta=\frac{1}{\chi_{1}}-\frac{1}{\chi_{2}}
$$

and (3.35) is now

$$
x=\frac{\sqrt{u}}{1+\sqrt{v}}, \quad \cosh \beta=\frac{1-\sqrt{v}}{\sqrt{u}}, \quad \text { with } \quad \sqrt{u} \leq 1-\sqrt{v} .
$$

To derive the leading behavior of the EMI in such a limit, we would need to study $x$ and $\cosh \beta$ for $L \gg R_{A}, R_{B}$ using eqs. (3.37) and (3.12). The relevant leading expressions are thus (see for instance section (2.2) of [7])

$$
x \sim \frac{2 R_{A} R_{B}}{L^{2}}, \quad \text { and } \quad \cosh \beta \sim\left[2\left(n_{A} \cdot l\right)\left(n_{B} \cdot l\right)-n_{A} \cdot n_{B}\right] .
$$

In the long distance limit $x \ll 1$ and $\cosh \beta \sim \mathcal{O}(1)$. As a consequence, the leading behavior of eq. (3.32) in this regime can be obtained by setting $x=0$ in the integrand, 
which makes the hypergeometric function equal to one, and replacing everywhere else the relations (3.39). This leads to

$$
\begin{aligned}
I_{\mathrm{EMI}} \stackrel{\left(L \gg R_{A}, R_{B}\right)}{=} & \frac{2^{5} \kappa_{(d)} \pi^{d-\frac{3}{2}}\left(\frac{d-2}{d-1}\right)}{2^{d-1} \Gamma\left(\frac{d-2}{2}\right) \Gamma\left(\frac{d-1}{2}\right)}\left(\frac{2 R_{A} R_{B}}{L^{2}}\right)^{d-1} \\
& \times\left[2\left(n_{A} \cdot l\right)\left(n_{B} \cdot l\right)-n_{A} \cdot n_{B}\right] \int_{0}^{1} \mathrm{~d} \xi\left(1-\xi^{2}\right)^{\frac{d-4}{2}} .
\end{aligned}
$$

The remaining integral can be trivially done,

$$
\int_{0}^{1} \mathrm{~d} \xi\left(1-\xi^{2}\right)^{\frac{d-4}{2}}=\frac{\sqrt{\pi}}{2} \frac{\Gamma\left(\frac{d-2}{2}\right)}{\Gamma\left(\frac{d-1}{2}\right)}
$$

leading to the final result presented in eq. (2.4) which, as mentioned above, agrees up to an undetermined overall constant - with the long-distance behavior of the mutual information dominated by a free fermion.

We can similarly study (3.36) (with an overall minus sign change due to the new relations (3.35)). In that case, the analogous leading expressions for $u$ and $v$ are

$$
u \sim \frac{16 R_{A}^{2} R_{B}^{2}}{L^{4}}+\mathcal{O}\left(L^{-5}\right), \quad \text { and } \quad v \sim 1-\frac{8 R_{A} R_{B}}{L^{2}}\left[2\left(n_{A} \cdot l\right)\left(n_{B} \cdot l\right)-n_{A} \cdot n_{B}\right]+\mathcal{O}\left(L^{-3}\right) .
$$

and plugging these into eq. (3.36) will lead to eq. (2.4).

Since $u$ and $v$ are in general independent variables, we can study other limits. For instance, we can take $u \rightarrow 0$ while leaving $v$ untouched, or take $v \rightarrow 1$ with $u$ arbitrary, among others. Let us mention the $u \rightarrow 0$ case with arbitrary $v$, as it turns out to have a simple analytic form. Indeed, in this limit the integral in (3.36) can be carried out explicitly, leading to

$$
\begin{aligned}
\int_{0}^{1} \mathrm{~d} \xi\left(1-\xi^{2}\right)^{\frac{d-4}{2}}{ }_{2} F_{1}\left[\frac{d}{2}, \frac{d-1}{2}, \frac{d+1}{2} ;\left(\frac{1-\sqrt{v}}{1+\sqrt{v}}\right)^{2} \xi^{2}\right] \\
=\frac{4(d-1) \sqrt{\pi} \Gamma\left(\frac{d-2}{2}\right)}{\Gamma\left(\frac{d+1}{2}\right)}{ }_{2} F_{1}\left[\frac{1}{2}, \frac{d}{2}, \frac{d+1}{2} ;\left(\frac{1-\sqrt{v}}{1+\sqrt{v}}\right)^{2}\right],
\end{aligned}
$$

and thus the EMI becomes

$$
I_{\mathrm{EMI}} \stackrel{u \rightarrow 0}{=} \frac{4 \pi^{d-1}(d-1)(d-2)}{2^{d-1}\left(\Gamma\left(\frac{d+1}{2}\right)\right)^{2}} \kappa_{(d)}\left(\frac{\sqrt{u}}{1+\sqrt{v}}\right)^{d-2}\left(\frac{1-\sqrt{v}}{1+\sqrt{v}}\right){ }_{2} F_{1}\left[\frac{1}{2}, \frac{d}{2}, \frac{d+1}{2} ;\left(\frac{1-\sqrt{v}}{1+\sqrt{v}}\right)^{2}\right] .
$$

Expression (3.44) can be compared directly with the leading contributing conformal block. For $u \rightarrow 0$ and $v$ arbitrary, this reads [45]

$$
G_{d-1,1}^{d}(u, v) \stackrel{u \rightarrow 0}{=} u^{\frac{d-2}{2}}(1-v){ }_{2} F_{1}\left[\frac{d}{2}, \frac{d}{2}, d ; 1-v\right],
$$


whose functional dependence coincides exactly with (3.44) after the use of some identities. ${ }^{6}$ Thus, comparing with the expected behavior of $I_{\mathrm{EMI}}(A, B)$ at long distances, this is

$$
\left.I_{\mathrm{EMI}}(A, B)\right|_{u \rightarrow 0}=\left.b_{d-1,1}^{\text {(ferm) }} G_{d-1,1}^{d}(u, v)\right|_{u \rightarrow 0},
$$

where $b_{d-1,1}^{\text {(ferm) }}$ was defined in eq. (3.6), we can read off the required value for $\kappa_{(d)}$. The result appears in eq. (2.5) above. Already at this point it is puzzling that the $u \rightarrow 0$ limit of (3.36) agrees exactly with the contribution of the leading conformal block away from $v \rightarrow 1$. In other words, it is surprising to find a perfect agreement away from the usual long-distance limit.

\subsubsection{Zero relative boost}

An important limiting case corresponds to $\cosh \beta=1$, which describes concentric spheres lying on the same space-like hyperplane - which we can take to be the $t=$ constant surface. In that case, we can write down a closed analytic expression for $I_{\mathrm{EMI}}(A, B)$ for all $d$. First, in that limit, the argument of the hypergeometric function in (3.32) becomes independent of the integration variable and one finds

$$
I_{\mathrm{EMI}}(A, B)=\frac{4 \pi^{d-1}(d-1)(d-2)}{2^{d-1} \Gamma\left[\frac{d+1}{2}\right]^{2}} \kappa_{(d)} x^{d-1}{ }_{2} F_{1}\left[\frac{d}{2}, \frac{d-1}{2}, \frac{d+1}{2} ; x^{2}\right],
$$

where $x$ can be determined in terms of the geometric parameters for disjoint spheres on the same hyperplane, using (3.37) and (3.12). For spheres on the same hyperplane it is convenient to define the vector that joins the spheres' centers,

$$
D \equiv L l+R_{B} n_{B}-R_{A} n_{A}
$$

since now, $n_{A} \cdot D=0, n_{B} \cdot D=0$ and $n_{A} \cdot n_{B}=-1$. Trading $L$ for $D, \chi_{1}$ and $\chi_{2}$ take the form

$$
\chi_{1}=\frac{4 R_{A} R_{B}}{|D|^{2}-\left(R_{A}-R_{B}\right)^{2}}, \quad \chi_{2}=\frac{4 R_{A} R_{B}}{|D|^{2}-\left(R_{A}+R_{B}\right)^{2}},
$$

one can check using (3.37) that $\cosh \beta=1$ as we wanted, and

$$
x=\frac{2 R_{A} R_{B}}{|D|^{2}-R_{A}^{2}-R_{B}^{2}} .
$$

Plugging this expression into (3.48) gives us the EMI mutual information as a function of the physical parameters.

As described at the beginning of the section, we would like to subtract from this expression the contribution of the conformal block associated to the spin 1 conserved current

\footnotetext{
${ }^{6}$ We found the following identity numerically,

$$
4\left(\frac{2}{1+\sqrt{v}}\right)^{d-2}\left(\frac{1-\sqrt{v}}{1+\sqrt{v}}\right){ }_{2} F_{1}\left[\frac{1}{2}, \frac{d}{2}, \frac{d+1}{2} ;\left(\frac{1-\sqrt{v}}{1+\sqrt{v}}\right)^{2}\right]=(1-v)_{2} F_{1}\left[\frac{d}{2}, \frac{d}{2}, d ; 1-v\right] .
$$
}

Perhaps this follows from standard hypergeometric identities. Otherwise, it would be interesting to prove it rigorously. 
made out of free fermions, namely, the term $b_{d-1,1}^{(\text {ferm })} G_{d-1}^{d}(u, v)$. The limit considered above, $\cosh \beta=1$, corresponds in the $u$ and $v$ variables to the case in which

$$
\sqrt{u}=1-\sqrt{v},
$$

which follows from (3.38). However, it turns out that in the CFT literature these conformal blocks are often written in terms of yet another set of variables (parameters), $\{y, z\}$ which are defined via the relations

$$
u=y z, \quad \text { and } \quad v=(1-y)(1-z) .
$$

In terms of these variables, the constraint equation (3.52) implies

$$
y=z
$$

This is called the "diagonal limit" of the conformal blocks [46]. In such limit, $u$ and $v$ become simple functions of $z$

$$
u(z)=z^{2}, \quad \text { and } \quad v(z)=(1-z)^{2} .
$$

Now, a close formula for a conformal block of arbitrary scaling dimension and spin was derived in [47]. For $\Delta=d-1$ and $J=1$ this reads

$$
G_{d-1,1}^{d}(u(z), v(z)) \equiv G_{d-1,1}^{d}(z)=\frac{2-z}{2 z}\left(\frac{z^{2}}{1-z}\right)^{\frac{d}{2}}{ }_{2} F_{1}\left[1, \frac{d}{2}, \frac{d+1}{2} ; \frac{z^{2}}{4(z-1)}\right]
$$

Hence, our goal is then to subtract from (3.48) the function

$$
b_{d-1,1}^{\text {(ferm) }} G_{d-1,1}^{d}(z)=2^{\left[\frac{d}{2}\right]+1} \frac{\sqrt{\pi} \Gamma(d)}{4^{d} \Gamma\left(d+\frac{1}{2}\right)} \frac{2-z}{2 z}\left(\frac{z^{2}}{1-z}\right)^{\frac{d}{2}}{ }_{2} F_{1}\left[1, \frac{d}{2}, \frac{d+1}{2} ; \frac{z^{2}}{4(z-1)}\right],
$$

for which we need to rewrite eq. (3.48) as a function of $z$. The relation between $x$ and $z$ can be easily derived from (3.38) and yields

$$
x=\frac{\sqrt{u(z)}}{1+\sqrt{v(z)}}=\frac{z}{2-z} .
$$

Plugging this into (3.38) and using the calibrated value of the parameter $\kappa_{(d)}(2.5)$, we obtain

$$
I_{\mathrm{EMI}}(A, B)=2^{\left[\frac{d}{2}\right]+1} \frac{\sqrt{\pi} \Gamma(d)}{4^{d} \Gamma\left(d+\frac{1}{2}\right)} \frac{2-z}{2 z}\left(\frac{z^{2}}{1-z}\right){ }_{2}^{\frac{d}{2}} F_{1}\left[1, \frac{d}{2}, \frac{d+1}{2} ; \frac{z^{2}}{4(z-1)}\right],
$$

which is identical to $b_{d-1,1}^{\text {(ferm) }} G_{d-1,1}^{d}(z)$. This is a rather surprising result. For spherical regions, the EMI exactly equals the conformal block contribution of a spin 1 conserved current made out of a free fermion field. In other words, no other operators contribute to the sum in the r.h.s. of (3.4), and the iterative process mentioned at the beginning of the section is completed after the very first step. 


\subsubsection{General case for particular dimensions}

At this point it is obvious that in the most general case we must have the equality

$$
I_{\mathrm{EMI}}(A, B)=b_{d-1,1}^{\text {(ferm) }} G_{d-1,1}^{d}(u, v),
$$

and indeed, there is an elegant way to prove this equivalence using a smeared representation of the so-called OPE blocks. The reader interested in seeing such derivation can find it in appendix B.

It is instructive to write down closed-form expressions for each conformal block by explicitly computing the integral in (3.32) for the various dimensions. We rewrite the formula here for convenience

$$
\begin{aligned}
I_{\mathrm{EMI}}(A, B)= & \frac{2^{d} \Gamma\left[\frac{d-1}{2}\right] b_{d-1,1}^{(\text {ferm })}}{\sqrt{\pi} \Gamma\left[\frac{d-2}{2}\right]} x^{d-1} \cosh \beta \\
& \times \int_{0}^{1} \mathrm{~d} \xi\left(1-\xi^{2}\right)^{\frac{d-4}{2}}{ }_{2} F_{1}\left[\frac{d}{2}, \frac{d-1}{2}, \frac{d+1}{2} ; x^{2}\left(1+\sinh ^{2} \beta \xi^{2}\right)\right],
\end{aligned}
$$

where we have included explicitly the factor $b_{d-1,1}^{\text {(ferm) }}$ to facilitate comparison with the conformal blocks. Its dependence on the physical parameters $R_{A}, R_{B}, L$ and the unit vectors $n_{A}, n_{B}$ and $l$ can be obtained from eq. (3.37) and the definitions (3.12). Let us study now the above expression for $d=3,4,5,6$.

Three dimensions. In this case, the integral in eq. (3.61) gives

$$
\int_{0}^{1} \frac{\mathrm{d} \xi}{\sqrt{1-\xi^{2}}}{ }_{2} F_{1}\left[1, \frac{3}{2}, 2 ; x^{2}\left(1+\sinh ^{2} \beta \xi^{2}\right)\right]=\frac{2}{x^{2}}\left[\frac{\Pi\left(-\sinh ^{2} \beta \mid \frac{\sinh ^{2} \beta x^{2}}{1-x^{2}}\right)}{\sqrt{1-x^{2}}}-\frac{\pi}{2 \cosh \beta}\right],
$$

where $\Pi(n \mid m)$ is the complete elliptic integral of third kind $^{7}$ and its second argument in $(3.62)$ is bounded as

$$
0 \leq \frac{\sinh ^{2} \beta x^{2}}{1-x^{2}} \leq 1
$$

Hence, the result for the mutual information is

$$
I_{\mathrm{EMI}}(A, B)=\frac{16}{\pi} b_{2,1}^{\text {(ferm) }}\left[\frac{\cosh \beta}{\sqrt{1-x^{2}}} \Pi\left(-\sinh ^{2} \beta \mid \frac{\sinh ^{2} \beta x^{2}}{1-x^{2}}\right)-\frac{\pi}{2}\right] .
$$

\footnotetext{
${ }^{7}$ We adopt the following conventions for the elliptic integrals used in this paper. The elliptic integral of first kind $\mathrm{K}(n)$, second kind $\mathrm{E}(m)$ and third kind $\Pi(n \mid m)$ are defined as:

$$
\mathrm{K}(n)=\int_{0}^{\frac{\pi}{2}} \frac{\mathrm{d} \theta}{\sqrt{1-n \sin ^{2} \theta}}, \quad \mathrm{E}(m)=\int_{0}^{\frac{\pi}{2}} \sqrt{1-m \sin ^{2} \theta} \mathrm{d} \theta,
$$

and

$$
\Pi(n \mid m):=\int_{0}^{\frac{\pi}{2}} \frac{\mathrm{d} \theta}{\left(1-n \sin ^{2} \theta\right) \sqrt{1-m \sin ^{2} \theta}} .
$$


To compare with the CFT literature it is convenient to write the expression in terms of the $\{y, z\}$ variables defined in (3.53). Here we write each term appearing in (3.66) instead. We have ${ }^{8}$

$$
\begin{aligned}
\sinh ^{2} \beta & =\frac{2-y-z-2 \sqrt{(1-y)(1-z)}}{y z}, \quad \frac{\sinh ^{2} \beta x^{2}}{1-x^{2}}=\frac{(2-y-z-2 \sqrt{(1-y)(1-z)})^{2}}{(y-z)^{2}}, \\
\frac{\cosh \beta}{\sqrt{1-x^{2}}} & =\frac{y z-y-z}{|y-z|} \sinh \beta .
\end{aligned}
$$

In $d=3$ there is no general closed-form expression for the corresponding conformal block to compare with, although the interested reader can find an integral representation in [45], which can be shown to be equivalent to (3.68) for $\Delta=2$ and $J=1$. For comparison purposes, however, we prefer to turn the series representation of [48] for $d=3$ into an integral representation - see eq. (C.13) below - and numerically compare the results of both expressions. We find a perfect match.

Four dimensions. In this case, the relevant integral can also be done explicitly and its answer turns out to be given in terms of elementary functions, namely,

$$
\int_{0}^{1} \mathrm{~d} \xi_{2} F_{1}\left[2, \frac{3}{2}, \frac{5}{2} ; x^{2}\left(1+\sinh ^{2} \beta \xi^{2}\right)\right]=\frac{3}{2 x^{3}}\left[\frac{\tanh ^{-1}\left[\frac{\sinh \beta x}{\sqrt{1-x^{2}}}\right]}{\sinh \beta \sqrt{1-x^{2}}}-\frac{\tanh ^{-1}[x \cosh \beta]}{\cosh \beta}\right] .
$$

Therefore, the mutual information is

$$
I_{\mathrm{EMI}}(A, B)=12 b_{3,1}^{\text {(ferm) }}\left[\frac{\cosh \beta}{\sinh \beta \sqrt{1-x^{2}}} \tanh ^{-1}\left[\sqrt{\frac{\sinh ^{2} \beta x^{2}}{1-x^{2}}}\right]-\tanh ^{-1}[x \cosh \beta]\right] .
$$

This result can be expressed more simply in terms of the $\{y, z\}$ variables. We write the various pieces explicitly,

$$
\begin{aligned}
\tanh ^{-1}\left[x \sqrt{1+\cosh ^{2} \beta}\right] & =\frac{1}{4} \log [(1-y)(1-z)] \\
\tanh ^{-1}\left[\sqrt{\frac{\sinh ^{2} \beta x^{2}}{1-x^{2}}}\right] & =\frac{1}{2} \log \left(\frac{2-y-z+|y-z|}{2 \sqrt{(1-y)(1-z)}}\right) .
\end{aligned}
$$

Using eq. (3.68), and after some simplifications we get for the mutual information:

$$
I_{\mathrm{EMI}}(A, B)=3 b_{3,1}^{\text {(ferm) }}\left[\frac{y z-y-z}{(y-z)} \log \left(\frac{1-z}{1-y}\right)-\log [(1-y)(1-z)]\right] .
$$

${ }^{8}$ For $0 \leq y, z \leq 1$ the expressions can be written as

$$
\sinh ^{2} \beta=\frac{(\sqrt{1-y}-\sqrt{1-z})^{2}}{y z}, \quad \frac{\sinh ^{2} \beta x^{2}}{1-x^{2}}=\frac{(\sqrt{1-y}-\sqrt{1-z})^{4}}{(y-z)^{2}} .
$$

However, in some applications it is important not to make such assumption since one would need the expression to be valid in the whole complex plane. 
Indeed, for $d=4$, earlier work on conformal blocks provided a general formula for the conformal block associated to any operator with arbitrary scaling dimension and spin. Evaluating $\Delta=d-1$ and $J=1$ in the general formula — see e.g., eq. (7.1) of [49] — one gets

$$
G_{3,1}^{4}(y, z)=3\left[\frac{y z-y-z}{(y-z)} \log \left(\frac{1-z}{1-y}\right)-\log [(1-y)(1-z)]\right]
$$

in perfect agreement with our results.

Five dimensions. In this case one needs to work a bit harder to find a closed-form expression. However, the final answer can also be written compactly in terms of Elliptic integrals of the first, second and third kinds. The relevant integral is

$$
\begin{aligned}
& \int_{0}^{1} \mathrm{~d} \xi \sqrt{1-\xi^{2}}{ }_{2} F_{1}\left[2, \frac{5}{2}, 3 ; x^{2}\left(1+\sinh ^{2} \beta \xi^{2}\right)\right] \\
&=\frac{2}{3 x^{4} \cosh ^{4} \beta}\left[\pi \cosh \beta+\frac{2}{\sqrt{1-x^{2} \cosh ^{2} \beta}}\left\{\frac { \operatorname { c o s h } ^ { 2 } \beta } { \operatorname { s i n h } ^ { 2 } \beta } \left[\mathrm{E}\left(\frac{x^{2} \sinh ^{2} \beta}{x^{2} \cosh ^{2} \beta-1}\right)\right.\right.\right. \\
&\left.\left.\left.-\mathrm{K}\left(\frac{x^{2} \sinh ^{2} \beta}{x^{2} \cosh ^{2} \beta-1}\right)\right]-\Pi\left(\frac{\sinh ^{2} \beta}{\cosh ^{2} \beta} \mid \frac{x^{2} \sinh ^{2} \beta}{x^{2} \cosh ^{2} \beta-1}\right)\right\}\right],
\end{aligned}
$$

where K, E and $\Pi$ are the elliptic integrals of first, second and third kind - the conventions are described in eqs. (3.63) and (3.64). Since the above is a rather complicated formula, we checked that it reduces to the right expression for $\beta \rightarrow 0$. Our formula for the EMI in $d=5$ is therefore

$$
\begin{aligned}
& I_{\mathrm{EMI}}(A, B)=\frac{128}{3 \pi} \frac{b_{4,1}^{\text {(ferm) }}}{\cosh ^{3} \beta}\left[\pi \cosh \beta+\frac{2}{\sqrt{1-x^{2} \cosh ^{2} \beta}}\right. \\
& \left.\times\left\{\frac{\cosh ^{2} \beta}{\sinh ^{2} \beta}\left[\mathrm{E}\left(\frac{x^{2} \sinh ^{2} \beta}{x^{2} \cosh ^{2} \beta-1}\right)-\mathrm{K}\left(\frac{x^{2} \sinh ^{2} \beta}{x^{2} \cosh ^{2} \beta-1}\right)\right]-\Pi\left(\frac{\sinh ^{2} \beta}{\cosh ^{2} \beta} \mid \frac{x^{2} \sinh ^{2} \beta}{x^{2} \cosh ^{2} \beta-1}\right)\right\}\right],
\end{aligned}
$$

where the arguments of the Elliptic functions in terms of the $\{y, z\}$ variables have the form

$$
\begin{aligned}
\frac{\sinh ^{2} \beta}{\cosh ^{2} \beta} & =\frac{2-y-z-2 \sqrt{(1-y)(1-z)}}{2-y-z-2 \sqrt{(1-y)(1-z)}+y z}, \\
\frac{x^{2} \sinh ^{2} \beta}{x^{2} \cosh ^{2} \beta-1} & =-\frac{2-y-z-2 \sqrt{(1-y)(1-z)}}{4 \sqrt{(1-y)(1-z)}} .
\end{aligned}
$$

We can rewrite the other terms using the relations

$$
x=\frac{\sqrt{y z}}{1+\sqrt{(1-y)(1-z)}}, \quad \text { and } \quad \sinh ^{2} \beta=\frac{2-y-z-2 \sqrt{(1-y)(1-z)}}{y z}
$$

respectively. Similarly to the $d=3$ case, due to the lack of a closed-form expression for the corresponding conformal block, we numerically compare our result with the ones of appendix $\mathrm{C}$, finding perfect agreement. 
Six dimensions. Once again, the resulting integral can be carried out explicitly, leading to

$$
\begin{aligned}
\int_{0}^{1} \mathrm{~d} \xi\left(1-\xi^{2}\right) & { }_{2} F_{1}\left[\frac{5}{2}, 3, \frac{7}{2} ; x^{2}\left(1+\sinh ^{2} \beta \xi^{2}\right)\right] \\
=\frac{5}{8 x^{5}} & {\left[\frac{1-2 \sinh ^{2} \beta+x^{2}\left(1-3 \sinh ^{2} \beta\right)}{\sinh ^{3} \beta\left(1-x^{2}\right)^{\frac{3}{2}}} \tanh ^{-1}\left[\frac{\sinh \beta x}{\sqrt{1-x^{2}}}\right]\right.} \\
& \left.-2 \frac{\tanh ^{-1}[x \cosh \beta]}{\cosh \beta}-\frac{x}{\left(1-x^{2}\right) \sinh ^{2} \beta}\right] .
\end{aligned}
$$

Using relations (3.71), plus

$$
\cosh \beta\left[\frac{1-2 \sinh ^{2} \beta+x^{2}\left(1-3 \sinh ^{2} \beta\right)}{\sinh ^{3} \beta\left(1-x^{2}\right)^{\frac{3}{2}}}\right]=-\frac{2(y+z-y z)\left(y^{2}(1+z)+z^{2}(1+y)-4 y z\right)}{|y-z|^{3}},
$$

and

$$
\frac{\cosh \beta x}{\left(1-x^{2}\right) \sinh ^{2} \beta}=\frac{y z(y+z-y z)}{(y-z)^{2}},
$$

we can write down the expression for the mutual information in terms of the $\{y, z\}$ variables explicitly,

$$
\begin{aligned}
I_{\mathrm{EMI}}(A, B)=15 b_{5,1}^{\text {(ferm) }}[ & \frac{(y z-y-z)\left(y^{2}(1+z)+z^{2}(1+y)-4 y z\right)}{(y-z)^{3}} \log \left(\frac{1-z}{1-y}\right) \\
& \left.+\frac{2 y z(y z-y-z)}{(y-z)^{2}}-\log [(1-y)(1-z)]\right] .
\end{aligned}
$$

The result for the general conformal block for $d=6$ was originally derived in [45] - e.g., see their eq. (5.14). In the case of interest here, their result for $G_{5,1}^{6}(y, z)$ reduces to $I_{\mathrm{EMI}}(A, B) / b_{5,1}^{\text {(ferm) }}$, in perfect agreement with our general result.

\section{Free fermion at long distances for arbitrary regions}

As mentioned earlier, for the EMI model the mutual information of two entangling regions $A, B$ is given, in the long-distance limit, by the expression

$$
I_{\mathrm{EMI}}(A, B)=4(d-1)(d-2) \kappa_{(d)} \frac{\operatorname{vol}(A) \cdot \operatorname{vol}(B)}{r^{2(d-1)}}+\mathcal{O}\left(r^{-2 d+1}\right) .
$$

Here, $\kappa_{(d)}$ is the usual constant characteristic of the model, which is therefore completely independent of the geometry of $A$ and $B$. The expression analogous to eq. (4.1) for a free fermion reads [29]

$$
I_{\text {ferm }}(A, B)=g(A, B) \cdot \frac{\operatorname{vol}(A) \cdot \operatorname{vol}(B)}{r^{2(d-1)}}+\mathcal{O}\left(r^{-2 d+1}\right),
$$

where $g(A, B)$ is a number which, in principle, may depend on the geometry of the regions. Given the match in the scaling, it is natural to wonder whether the free fermion actually 
coincides exactly with the EMI in this regime. Where in this case, the function $g(A, B)$ would in fact be given by a fixed constant, independent of the geometric details of $A$ and $B$.

In order to test this possibility, we will compute here the mutual information for different shapes for a free fermion. From eq. (2.3) we can analytically extract $g(A, B)$ in the case of two disk regions for the free fermion. We have

$$
\left.g(A, B)\right|_{\text {spheres }}=\frac{2^{\left[\frac{d}{2}\right]-1} \Gamma(d)\left(\Gamma\left(\frac{d+1}{2}\right)\right)^{2}}{\pi^{d-\frac{3}{2}} \Gamma\left(d+\frac{1}{2}\right)} .
$$

A useful expression for $g(A, B)$ was obtained in [29] in terms of the resolvent of the vacuum correlator of the Dirac field restricted to each region, $A$ and $B$. For the twopoint function $C(x, y) \equiv\left\langle\Psi(x) \Psi(y)^{\dagger}\right\rangle$, the resolvent is defined as $R(\beta) \equiv[C+\beta-1 / 2]^{-1}$. Then, for regions which are mirror symmetric of themselves with respect to the line which separates $A$ and $B$, we have

$$
\begin{aligned}
g(A, B) \operatorname{vol}(A) \cdot \operatorname{vol}(B)= & \frac{\left(\Gamma\left(\frac{d}{2}\right)\right)^{2}}{4 \pi^{d}} \int_{1 / 2}^{\infty} \mathrm{d} \beta(\beta-1 / 2) \\
& \times\left[\operatorname{tr}\left[\overline{R_{A}}(\beta) \overline{R_{B}^{2}}(\beta)+\overline{R_{B}}(\beta) \overline{R_{A}^{2}}(\beta)\right]-(\beta \leftrightarrow-\beta)\right],
\end{aligned}
$$

where $\overline{R_{A}}(\beta) \equiv \int_{x \in A} \int_{y \in A} R(\beta ; x, y)$ denotes sum over the spatial variables belonging to region $A$.

Using this formula we compute the coefficient $g(A, B)$ in $d=2+1$ in the lattice, and test the possible invariance with the shape of the regions. From eq. (4.3) we have

$$
\left.g(A, B)\right|_{\text {disks }} ^{d=3}=\frac{16}{15 \pi^{2}} .
$$

This will also allow us to test the precision of our numerical calculations.

The formula (4.4) can be exploited in the lattice as follows. First, let $C_{A, i j}^{\alpha \beta} \equiv\left\langle\psi_{i}^{\alpha} \psi_{j}^{\dagger \beta}\right\rangle$ be the correlator for lattice fermionic fields corresponding to $i, j=1, \ldots, N_{A}$ where $N_{A}$ is the number of lattice sites of $A$ and where we denoted spinorial indices by $\alpha, \beta=0,1$. Then, if we denote by $u_{A, j}^{\left(\lambda_{A}\right) \beta}$ the eigenvector of $C_{A, i j}^{\alpha \beta}$ corresponding to a given eigenvalue $\lambda_{A}$, we can write

$$
\sum_{j, \beta} C_{A, i j}^{\alpha \beta} u_{A, j}^{\left(\lambda_{A}\right) \beta}=\lambda_{A} u_{A, i}^{\left(\lambda_{A}\right) \alpha}, \quad C_{A, i j}^{\alpha \beta}=\sum_{\lambda_{A}} \lambda_{A} u_{A, i}^{\left(\lambda_{A}\right) \alpha} u_{A, j}^{\left(\lambda_{A}\right) \beta^{\dagger}}
$$

Inserting the second expression in eq. (4.4) and using

$$
\begin{aligned}
\int_{1 / 2}^{\infty} \mathrm{d} \beta(\beta-1 / 2)[ & \frac{1}{\left(\lambda_{A}+\beta-1 / 2\right)\left(\lambda_{B}+\beta-1 / 2\right)^{2}}+\frac{1}{\left(\lambda_{B}+\beta-1 / 2\right)\left(\lambda_{A}+\beta-1 / 2\right)^{2}} \\
& -(\beta \leftrightarrow-\beta)]=f\left(\lambda_{A}, \lambda_{B}\right)
\end{aligned}
$$

where

$$
f\left(\lambda_{A}, \lambda_{B}\right) \equiv \frac{1}{\left(\lambda_{A}-\lambda_{B}\right)} \log \left[\frac{\lambda_{A}\left(1-\lambda_{B}\right)}{\lambda_{B}\left(1-\lambda_{A}\right)}\right]
$$




\begin{tabular}{|c|c|c|c|c|c|}
\hline & Disks & Squares & Rect. $(2 L \times L)$ & Rect. $(4 L \times L)$ & Rect. $(6 L \times L)$ \\
\hline$g(A, B) \times \frac{15 \pi^{2}}{16}$ & 0.994 & 0.997 & 0.938 & 0.862 & 0.840 \\
\hline
\end{tabular}

Table 1. Continuum values of the coefficient $g(A, B)$ normalized by the analytic result corresponding to a pair of disks, $16 /\left(15 \pi^{2}\right)$, obtained in the lattice for different pairs of identical regions.

we find the formula

$g(A, B) \operatorname{vol}(A) \cdot \operatorname{vol}(B)=\frac{1}{16 \pi^{2}} \sum_{\lambda_{A}, \lambda_{B}} f\left(\lambda_{A}, \lambda_{B}\right)\left|v_{B}^{\left(\lambda_{B}\right)^{\dagger}} \cdot v_{A}^{\left(\lambda_{A}\right)}\right|^{2}$, where $v_{A}^{\left(\lambda_{A}\right) \alpha} \equiv \sum_{i} u_{A, i}^{\left(\lambda_{A}\right) \alpha}$,

and, for fixed $\lambda_{A}$ and $\lambda_{B}, v_{B}^{\left(\lambda_{B}\right)^{\dagger}} \cdot v_{A}^{\left(\lambda_{A}\right)}$ denotes a scalar product between two-component vectors corresponding to the spinorial indices. In words, given the correlators matrix corresponding to each region, we first obtain its eigenvalues and eigenvectors. Using the former we can evaluate the function $f\left(\lambda_{A}, \lambda_{B}\right)$ for every pair of eigenvectors. Then, for each spinorial index $\alpha=0,1$, we sum over the lattice sites of each region in order to obtain the $v^{(\lambda), \alpha}$. Finally, we use eq. (4.9) to evaluate $g(A, B) .^{9}$

The three-dimensional lattice Hamiltonian for the free fermion reads

$$
H=-\frac{i}{2} \sum_{n, m}\left[\left(\psi_{m, n}^{\dagger} \gamma^{0} \gamma^{1}\left(\psi_{m+1, n}-\psi_{m, n}\right)+\psi_{m, n}^{\dagger} \gamma^{0} \gamma^{2}\left(\psi_{m, n+1}-\psi_{m, n}\right)\right)-\text { h.c. }\right],
$$

and the vacuum-state correlators are given in this case by [29]

$$
C_{(n, k),(j, l)}=\frac{1}{2} \delta_{n, j} \delta_{k l}-\int_{-\pi}^{\pi} \mathrm{d} x \int_{-\pi}^{\pi} \mathrm{d} y \frac{\sin (x) \gamma^{0} \gamma^{1}+\sin (y) \gamma^{0} \gamma^{2}}{8 \pi^{2} \sqrt{\sin ^{2} x+\sin ^{2} y}} e^{i(x(n-j)+y(k-l))},
$$

where here we used subindices $(i, j)$ to denote coordinates $x, y$ in the square lattice.

The idea is then to consider fixed shapes in the lattice and evaluate $g(A, B)$ as we increase the number of points. Performing inverse-power-law fits to the data - e.g., $\left\{1 / x^{3}, 1 / x^{2}, 1 / x, 1\right\}$ - we can read off the continuum results from the $\mathcal{O}(1)$ coefficients. In table 1 we present the results obtained for identical pairs of disks, squares, and rectangles of side-lengths: $2 L \times L, 4 L \times L$ and $6 L \times L$, respectively. As we can see, the numerical result obtained for the disks is very close to the analytic one appearing in eq. (4.3). Interestingly, the result for a pair of squares is also remarkably similar to the disks result. However, as we deform the squares and replace them with increasingly thinner rectangles, the coefficient decreases, clearly differing from the disks result.

In fact, the limit of very thin parallel plates with area $\mathcal{A}$ can be treated analytically in any dimension. In this case, we have a limit of translational invariance in the direction parallel to the plates. Consequently, the eigenvectors of the correlator decompose as

$$
u_{A}^{\lambda}(x) \sim \mathcal{A}^{-1 / 2} e^{i \vec{k}_{\|} \vec{x}_{\|}} \phi_{\lambda, \vec{k}_{\|}}\left(x_{\perp}\right) .
$$

\footnotetext{
${ }^{9}$ The analogous expression for the long-distance coefficient of the MI for scalar fields was obtained in [50]. For fermions, the same formula (4.9) applies in the lattice without correction for fermion doubling. The reason is that the eigenvectors are averaged over position and this eliminates the doubling modes which have large momentum $\sim \pi$ in the Brillouin zone.
} 
Upon averaging over the region all vectors with $\vec{k}_{\|} \neq 0$ do not contribute. Only the constant eigenvectors in the parallel directions contribute. On these, the correlator has the same effect as the correlator of a $d=2$ dimensional field, since the integration imposes zero parallel momentum in the parallel direction,

$$
\int \mathrm{d}^{d-2} x_{\|} C(\vec{x})=\int \mathrm{d}^{d-2} x_{\|} \int \frac{\mathrm{d}^{d} p}{(2 \pi)^{d}} \frac{e^{-i p x}}{i \not p} \gamma^{0}=\int \frac{\mathrm{d}^{2} p}{(2 \pi)^{2}} \frac{e^{-i p x}}{i \not p} \gamma^{0}=C_{2}\left(x^{1}\right),
$$

where, however, the spinorial representation is still of dimension $2^{\left[\frac{d}{2}\right]}$. The problem then collapses to a two dimensional problem of the mutual information of $2^{\left[\frac{d}{2}\right]-1}$ fermions for well separated intervals. Each $d=2$ fermion has $g(A, B)=1 / 3$. We then obtain from (4.4)

$$
\left.g(A, B)\right|_{\text {plates }}=\frac{\left(\Gamma\left(\frac{d}{2}\right)\right)^{2} 2^{\left[\frac{d}{2}\right]-1}}{3 \pi^{d-2}} .
$$

Therefore, in any dimensions we have

$$
\frac{\left.g(A, B)\right|_{\text {plates }}}{\left.g(A, B)\right|_{\text {spheres }}}=\frac{\pi \Gamma\left(\frac{d}{2}\right) \Gamma\left(d+\frac{1}{2}\right)}{32^{d-1}\left(\Gamma\left(\frac{d+1}{2}\right)\right)^{3}} .
$$

To compare with the numerical calculation above for rectangles, this gives for $d=3$ a ratio of $\sim 0.771$. The results in table 1 asymptotically approach this value as the rectangles become thinner. The ratio (4.15) is always less than 1 and decreases with dimension.

Hence, we conclude that the long-distance limit of the free fermion mutual information differs from the EMI one, as the latter depends on the geometry of the entangling regions exclusively through the product of volumes, whereas the former includes an additional shape-dependent function.

\section{Is the EMI the mutual information of a CFT?}

We have seen that the conjectural EMI theory must contain a fermion with dimension $(d-1) / 2$ as a lowest-dimensional operator. We have also shown that the long-distance leading term of the free fermion and EMI models do not coincide, while the EMI gives exactly the leading conformal block to the MI of the free fermion for spheres. We now show this is not compatible with the EMI model being the MI of a fixed CFT.

First, recall that if a QFT contains a field $\psi$ with the two-point function of a free field, then this field has to be free, that is, all its correlators satisfy Wick's theorem [1]. The key of the argument is that the two-point correlator satisfies a local equation. In consequence, the field operator itself must satisfy the free equation of motion. For example, for a field having the two-point function of the free fermion field, writing $u(x)=\gamma^{\mu} \partial_{\mu} \psi(x)$, we get $\left\langle 0\left|u^{\dagger}(x) u(y)\right| 0\right\rangle=0$. From this, the smeared operator $u_{\alpha}=\int \mathrm{d} x \alpha(x) u(x)$, satisfies $\left.\left|u_{\alpha}\right| 0\right\rangle\left.\right|^{2}=0 \Longrightarrow u_{\alpha}|0\rangle=0$, and since no local operator can annihilate the vacuum, $\gamma^{\mu} \partial_{\mu} \psi(x)=u(x)=0$. For a field satisfying the free equation of motion, the usual decomposition into positive and negative frequencies can be done, and the usual numeric 
commutator (anti-commutator) follows [1]. The c-number commutator implies Wick's theorem. This determines all correlators of the field with itself. Further, in the Hilbert space generated by acting with the field polynomials on the vacuum, the only other field operators that are mutually local (commuting or anticommuting at spacelike distances) with $\psi$ are polynomials on $\psi$ and its derivatives [51]. This means that the free field decouples from the rest of the theory and we have a tensor product of theories and Hilbert spaces

$$
\mathcal{T}_{\text {EMI }}=\mathcal{T}_{1} \otimes \mathcal{T}_{\psi}, \quad \mathcal{H}_{\text {EMI }}=\mathcal{H}_{1} \otimes \mathcal{H}_{\psi} .
$$

As a consequence of this decoupling, it is not difficult to see that the EMI mutual information cannot correspond to a CFT. First, the leading long-distance contribution for any shapes is dominated by the leading primary operator contribution to the OPE of the Rényi operators, which here is a free fermion field. The coefficient of this contribution depends on the modular flow of the regions acting on the fermion field. This modular flow is not universal for non-spherical regions and depends on the operator content of the full theory. However, as the fermion field decouples, the modular flow acting on the fermion field has to be the same as the flow of the free fermion theory. This gives the same coefficient for the long-distance contribution for the EMI and the free fermion for any shapes. This contradicts the results of section 4 .

Another important consequence of this decoupling is that eq. (5.1) would imply

$$
I_{\mathrm{EMI}}(A, B)=I_{1}(A, B)+I_{\psi}(A, B),
$$

and therefore

$$
I_{\mathrm{EMI}}(A, B) \geq I_{\psi}(A, B)
$$

for any $A, B$. The coefficient of the EMI model has to be adjusted to match the longdistance contribution of the free fermion for balls since $I_{1}(A, B)$ falls to zero faster at large distances. However, with this calibration, (5.3) is not violated for the contributions to the mutual information we have studied so far. Then, it does not give a different argument for the impossibility of the EMI model.

Another startling feature of EMI as a MI of a CFT is that for two spheres it consists entirely of a single conformal block of spin 1 and dimension $d-1$. This same feature holds for a free fermion in $d=2$. In that case, it is the result of a precise cancellation between the contributions of different primary fields in the replicated theory. We turn now to analyze whether this is possible for $d>2$.

\section{Can EMI represent a limit of CFTs?}

No fixed QFT can have a MI described by the EMI model for $d>2$. However, this result by itself does not imply that the EMI model has no physical meaning. To see this in perspective, we could think of applying the same ideas to extract the QFT from the mutual information for holographic EE [11]. The bulk minimal area in AdS is known to provide a formula for the entropy whose MI satisfies all the axioms (1.3)-(1.9), plus conformal invariance. However, if we try to bootstrap the holographic formula to obtain information 
on the operator content, we immediately encounter a problem: the long-distance MI is trivial since there are phase transitions that make it vanish for a long enough distance. A naive application of the same principles we have applied to the EMI model would lead to the conclusion that there are no operators in the theory, and that the Ryu-Takayanagi formula is inconsistent. Of course, as it is well known, the area term represents only the leading $N^{2}$ term in the holographic entropy, and there are subleading $N^{0}$ terms that do not vanish at large distances. They are given by the mutual information of free fields in the bulk entangling wedges [15]. Therefore, from the knowledge of the Ryu-Takayanagi term alone we cannot infer the physical validity of the model as the EE of a QFT comparing with the expected result for a fixed QFT.

This highlights a difference in the nature of the analysis of the validity of an entropy (or mutual information) function as compared to, e.g., checking the validity of a system of correlation functions. The reason is precisely that entropy is a quantity that can be defined universally for any QFT. There are several ways in which an entropy function satisfying (1.3)(1.9) can be obtained from valid QFTs but which do not represent the entropies of any QFT. In particular, the system of requirements (1.3)-(1.9) do not fix a normalization of the entropy, and this is related to the fact that any linear combination with positive coefficients of entropies of two theories will also satisfy the requirements. This is justified by the fact that entropies for a tensor product of theories are the sum of the corresponding entropies. Moreover, if we have a set of QFTs determined by a parameter, we can take limits on this parameter and the limit of the entropy will also satisfy the requirements. This parameter may be a coupling constant or some other parameter like the number $N$ of colors, as in large- $N$ models. Therefore, there may be many solutions to the constraints that do not represent actual QFTs but specific limits of QFTs. The holographic EE is one of these limits.

A natural question is if the EMI model represents some limit of CFTs. The main argument of the preceding section relies on the coefficient of long-distance mutual information for regions of arbitrary shape. These coefficients depend on the modular flow of the theory, which is not universal for nonspherical regions and depends on the full operator content. Then, with our present rather incomplete knowledge about modular Hamiltonians, it is not clear that the argument still holds for limits of theories. In taking this limit, we cannot discard from the outset having a set of fermions taking a free limit while the modular flow producing significant persistent differences in the coefficient with respect to the decoupled free fermion field.

However, we will answer the question of whether or not the EMI is a limit of QFT's in the negative. To do so, we turn attention again to spheres, where the modular flow is universal.

\subsection{Further analysis of the free fermion MI for spheres}

As the EMI contains a free fermion (as a limit), it must contain other contributions of the same field. In the long-distance limit, the leading such contribution comes, in the replica trick, from the field $\psi$ in one copy and the derivative $\partial \psi$ in another copy. Let us determine this operator with more precision. 
The OPE of a twist operator for the Rényi entropy of integer order $n$ for a sphere is of the form

$$
\tau^{(n)}=\sum C_{\alpha}^{(n)} \mathcal{O}^{\alpha},
$$

where the sum is over all local operators in the $n$-replicated theory, $\alpha$ are Lorentz indices, and the coefficients depend only on the theory and the geometric features of the sphere. Namely, the tensor structure of $C_{\alpha}^{(n)}$ can only depend on the time-like unit vector $n_{\mu}$ determining the orientation of the sphere in space-time, and $g_{\mu \nu}$. The leading long-distance term comes from the two copy operator (to simplify the notation we call 1,2 the two copies, which can be any pair of copies of the $n$ replicas)

$$
V^{\mu}=\bar{\psi}_{1} \gamma^{\mu} \psi_{2}-\bar{\psi}_{2} \gamma^{\mu} \psi_{1}
$$

This gives a contribution $\sim n_{\mu} V^{\mu}$ to $\tau^{(n)}$. The minus sign in (6.2) comes from chargeconjugation invariance. Other Dirac matrix structures do not contribute because of parity invariance or because they form antisymmetric tensors whose contraction with products of $n^{\mu}$ or the metric vanishes $[7,25]$.

The above operator has dimension $d-1$. The next operator comes from adding a derivative to the fermion bilinear, with dimension $d$. The descendant field

$$
\partial_{\alpha} V^{\mu}=\left(\partial_{\alpha} \bar{\psi}_{1}\right) \gamma^{\mu} \psi_{2}+\bar{\psi}_{1} \gamma^{\mu} \partial_{\alpha} \psi_{2}-\left(\partial_{\alpha} \bar{\psi}_{2}\right) \gamma^{\mu} \psi_{1}-\bar{\psi}_{2} \gamma^{\mu} \partial_{\alpha} \psi_{1}
$$

is already included in the first conformal block studied in section 3 . We recall that this conformal block contribution to the mutual information exactly coincides with the EMI for spheres. Then, we consider other bilinear operators with a single derivative that forms independent primary operators. Again, charge conjugation, parity, and the fact that this operator cannot have an antisymmetric structure to give a non-vanishing contribution leaves as the only possibility the two-copy operator

$$
V_{\alpha \mu}=\frac{1}{2}\left[\left(\partial_{\alpha} \bar{\psi}_{1}\right) \gamma_{\mu} \psi_{2}-\bar{\psi}_{1} \gamma_{\mu} \partial_{\alpha} \psi_{2}+\left(\partial_{\alpha} \bar{\psi}_{2}\right) \gamma_{\mu} \psi_{1}-\bar{\psi}_{2} \gamma_{\mu} \partial_{\alpha} \psi_{1}+(\alpha \leftrightarrow \mu)\right]
$$

Notice this operator is traceless due to the Dirac equation. It is a primary field of spin 2 (symmetric traceless two index tensor) of dimension $d$. For the free fermion, no other contribution of dimension less or equal to $d$ is possible if $d>2$. For $d=2$ we have the contribution of four fermions in four copies, with dimension $2(d-1)=2$. In $d=2$ this contribution exactly cancels the one from (6.4) because the full result coincides with the first conformal block. This will not be possible for $d>2$.

The two-point function has the form ${ }^{10}$

$$
\left\langle V_{\mu \nu}(0) V_{\alpha \beta}(x)\right\rangle \propto|x|^{-2 d} P_{\mu \nu, \delta_{1} \delta_{2}} I^{\delta_{1} \gamma_{1}}(\hat{x}) I^{\delta_{2} \gamma_{2}}(\hat{x}) P_{\gamma_{1} \gamma_{2}, \alpha \beta},
$$

where $P=P^{2}$ is the projector on the symmetric traceless tensors

$$
P_{\mu_{1} \mu_{2}, \nu_{1} \nu_{2}}=\frac{1}{2}\left(g_{\mu_{1} \nu_{1}} g_{\mu_{2} \nu_{2}}+g_{\mu_{1} \nu_{2}} g_{\mu_{2} \nu_{1}}-\frac{2}{d} g_{\mu_{1} \mu_{2}} g_{\nu_{1} \nu_{2}}\right)
$$

\footnotetext{
${ }^{10}$ The general structure of correlators of primary fields in a CFT consists of tensor products of the $I$ tensor projected on the adequate symmetry representation. Only one projector is enough in (6.5) since $P$ commutes with the tensor product of the $I$.
} 
and $I, I^{2}=1$, is the tensor

$$
I_{\mu \nu}(\hat{x})=g_{\mu \nu}-2 \hat{x}_{\mu} \hat{x}_{\nu} .
$$

The contribution to the Rényi operators for each sphere is then proportional to operators of the form $n^{\mu} n^{\nu} V_{\mu \nu}$, summed over all possible pairs of copies. The contribution to the mutual information to the lowest order has the same tensor structure of the correlation between these operators

$$
\Delta I(A, B) \propto n_{A}^{\mu} n_{A}^{\nu}\left\langle V_{\mu \nu}(0) V_{\alpha \beta}(L \hat{l})\right\rangle n_{B}^{\alpha} n_{B}^{\beta}
$$

The coefficient can be computed by the method of [7]. We relegate to appendix D the details of this calculation. We get

$$
\Delta I(A, B)=-\frac{\sqrt{\pi} \Gamma(d+1) 2^{\left[\frac{d}{2}\right]-1}}{\Gamma\left(d+\frac{3}{2}\right)} \frac{R_{A}^{d} R_{B}^{d}}{L^{2 d}}\left[\left(2\left(n_{A} \cdot l\right)\left(n_{B} \cdot l\right)-\left(n_{A} \cdot n_{B}\right)\right)^{2}-\frac{1}{d}\right] .
$$

Let us analyze whether this contribution to the free fermion can be canceled by some other contribution coming from additional fields. The lowest-dimensional contribution by a primary field of dimension $\Delta$ of the theory, different from the fermion, comes from the field in two copies and gives a dependence $L^{-4 \Delta}$. Then we must have $\Delta \leq d / 2$. Because of the unitarity bound, this leaves us with a scalar, a spin $1 / 2$ fermion, or a helicity one field (a completely antisymmetric field of $d / 2$ indices). This last possibility only exists for even dimensions, and, for $\Delta=d / 2$ saturates the unitarity bound. Then it must be a free field.

In fact, the tensor structure of the contribution (6.9) exactly matches the leading term of a helicity-1 field in any even dimensions and does not match the scalar or fermion contribution [7]. This eliminates the scalar and fermion as possibilities. However, in odd dimensions, there is no such conformal primary of dimension $d / 2$ and helicity equal to 1 . The only possibility to eliminate this contribution from the free fermion in the EMI model in odd dimensions would be to obtain it from descendant fields of a scalar. The contribution of the derivative of a free scalar could be present without the contribution of the scalar itself if the theory is generated by polynomials of $\partial_{\mu} \phi$. In this case, the theory is not conformally invariant and only scale invariant. The derivatives of the free scalar form the subalgebra stable under $\phi \rightarrow \phi+$ const. The contributions for spheres can be analyzed with the same methods. The contribution coming from fields $\partial_{\mu} \phi_{1} \partial_{\nu} \phi_{2}$ in two copies does not have the correct tensor structure. This is because, apart from a contribution independent from the orientation of the spheres, produced by $\partial_{\mu} \phi_{1} \partial^{\mu} \phi_{2}$, the tensor structure must be of the form

$$
n_{A}^{\mu} n_{A}^{\nu}\left\langle\partial_{\mu} \phi_{1}\left(r_{A}\right) \partial_{\nu} \phi_{2}\left(r_{A}\right) \partial_{\alpha} \phi_{1}\left(r_{B}\right) \partial_{\beta} \phi_{2}\left(r_{B}\right)\right\rangle n_{B}^{\alpha} n_{B}^{\beta} \sim \frac{1}{L^{2 d}}\left(d\left(n_{A} \cdot l\right)\left(n_{B} \cdot l\right)-\left(n_{A} \cdot n_{B}\right)\right)^{2} .
$$

This eliminates the possibility that the EMI can be produced by a limit of QFTs in odd dimensions.

For even dimensions, as the term (6.9) comes with a negative coefficient, we can cancel it by the contribution, with the adequate proportion, of a free helicity $h=1$ field, which 
has the same tensor structure $[7]$

$$
I_{h=1}(A, B) \sim \frac{\sqrt{\pi} \Gamma(d+1) \Gamma(d-1)}{\Gamma\left(d+\frac{3}{2}\right)\left(\Gamma\left(\frac{d}{2}\right)\right)^{2}} \frac{R_{A}^{d} R_{B}^{d}}{L^{2 d}}\left[\left(2\left(n_{A} \cdot l\right)\left(n_{B} \cdot l\right)-\left(n_{A} \cdot n_{B}\right)\right)^{2}-\frac{1}{d}\right],
$$

This would imply

$$
I_{\mathrm{EMI}}=I_{\text {fermion }}+b I_{h=1}+\cdots,
$$

where $b$ is the ratio (with positive sign) between the coefficients of (6.9) and (6.11),

$$
b=2^{\left[\frac{d}{2}\right]-1} \frac{\left(\Gamma\left(\frac{d}{2}\right)\right)^{2}}{\Gamma(d-1)} .
$$

In (6.12) we have again normalized the EMI to have the long-distance MI of the single Dirac fermion for spheres. Now, as the ellipsis in (6.12) represent other positive contributions to the MI of the spheres, we can check what happens for nearly-touching spheres, where we can extract the coefficient $k$ of the area term. From (6.12) we should have

$$
k_{\mathrm{EMI}} \geq k_{\text {fermion }}+b k_{h=1} .
$$

For $d=4$, using the results of section (2.2), and the ones for a Maxwell field $k_{\text {Maxwell }}=$ 0.0110 [52], we get a left hand side 0.0273 and for the right hand side 0.0325 . This is incompatible with (6.14). For higher even dimensions the number of field components in the totally antisymmetric field of $d / 2$ indices (the $h=1$ field) is $\left(\begin{array}{c}d \\ d / 2\end{array}\right)$, from which $2\left(\begin{array}{c}d-1 \\ d / 2+1\end{array}\right)$ are constraints. Half the rest of the components give momentum variables in the Hamiltonian formalism. The value of the $k$ for this field is then (see the similar calculation for a Maxwell field in [52])

$$
k_{h=1}=\frac{1}{2}\left(\left(\begin{array}{c}
d \\
d / 2
\end{array}\right)-2\left(\begin{array}{c}
d-1 \\
d / 2+1
\end{array}\right)\right) k_{\text {scalar }} .
$$

For dimension $\geq 6$ we can use the very good approximations [29]

$$
k_{\text {scalar }} \sim \frac{\Gamma\left(\frac{d-2}{2}\right)}{2^{d+2} \pi^{(d-2) / 2}}, \quad k_{\text {fermion }}=2^{\left[\frac{d}{2}\right]} k_{\text {scalar }} .
$$

Plugging this into (6.14) we see that for $d=6$ the left hand side is 0.00438 while the right hand side is 0.00459 . Then the inequality does not hold for $d=6$. However, it still holds for higher dimensions. This shows the mismatch between the EMI and the fermion for spheres cannot be remedied by adding other fields for $d=6$. For higher even dimensions the analysis should be extended further in the MI expansion. We will not attempt it here.

In conclusion, there is no way in which the EMI result could be the mutual information for a CFT, even considered as a limit of theories. This is because this limit of theories 
must contain a field (or many fields) with the two-point function of the free fermion. In that case, the next-to-leading-order term (6.9) for the mutual information must also be present, disregarding the particular limit of theories, because it depends only on the twopoint function and the universal form of the modular flow for spheres. The EMI does not contain such a term, and no such term could be canceled by other possible contributions.

\section{Discussion}

In this paper, we have shown that the EMI model is not compatible with the mutual information of a QFT (for $d>2$ ). This means that $I_{3} \equiv 0$ is inconsistent in relativistic QFT for $d>2$. Moreover, this shows that the known set of constraints for the mutual information in QFT is incomplete. Below we make some further comments on this result interpreted in terms of twist operators, the relation with the pinching property of the MI of free fields, and with reflection positivity inequalities satisfied by correlation functions.

Coherent twist operators. In the task of constructing viable entropy functions, a natural route would be to have an ansatz for the behavior of the Rényi twist operators. The simplest candidate would be to produce them out of free fields. This is, in fact, the way in which formulas analogous to the EMI model were proposed in [23]. Suppose we conjecture twist operators with the form of coherent operators

$$
\tau_{A}^{(n)}=e^{i \int_{A} \mathrm{~d} x \alpha_{A}^{(n)}(x) \phi^{(n)}(x)},
$$

where $\phi^{(n)}(x)$ is some generic Gaussian field (free or generalized free field), and $\alpha_{A}^{(n)}(x)$ is a function with support in the causal region $A$ and determined by the geometry of $A$. For example, it may have support only in the boundary of $A$, or in any Cauchy surface of $A$. In this latter case, it has to be conserved for different Cauchy surfaces. Under this assumption, we get for the mutual information a bilinear expression

$$
I(A, B)=\lim _{n \rightarrow 1}(n-1)^{-1} \log \left(\frac{\left\langle\tau_{A}^{(n)} \tau_{B}^{(n)}\right\rangle}{\left\langle\tau_{A}^{(n)}\right\rangle\left\langle\tau_{B}^{(n)}\right\rangle}\right)=\int_{A} \mathrm{~d} x \int_{B} \mathrm{~d} y \alpha_{A}(x) \alpha_{B}(y) G(x-y),
$$

for some geometric functions $\alpha_{A}, \alpha_{B}$, and where $G(x-y)$ is some two-point function. This form of the entropy immediately gives $I_{3}(A, B, C)=0$ for disjoint regions. Hence, this property seems naturally associated to twist operators which are exponentials of Gaussian fields.

However, in most cases for the functions $\alpha_{A}^{(n)}$ and the free fields $\phi^{(n)}$, the result for $I(A, B)$ is not monotonic. In fact, the only monotonic possibility for a MI with the structure (7.2) is the EMI function [16], with the precise form (1.11) or equivalently (1.12). In consequence, monotonicity imposes to the ansatz (7.1) a precise form, where the twist operators are exponentials of the flux of a current (local charge operator)

$$
\tau_{A}^{(n)} \sim e^{i \int_{\Sigma_{A}} \mathrm{~d} \sigma J_{\mu}^{(n)} \eta_{A}^{\mu}}
$$

but where the conserved current operator $J_{\mu}^{(n)}$ must be Gaussian. For $d=2$ this is the case of the Dirac current by bosonization. For $d>2$, this implies $J_{\mu}^{(n)}$ is a generalized free 
field (GFF). It is direct that evaluating the logarithm of the expectation values of these type of twist operators we get the expression (1.11) for the MI of the EMI model,

$$
I_{\mathrm{EMI}}(A, B) \propto\left\langle\left(\int_{\Sigma_{A}} \mathrm{~d} \sigma J_{\mu} \eta_{A}^{\mu}\right)\left(\int_{\Sigma_{B}} \mathrm{~d} \sigma J_{\mu} \eta_{B}^{\mu}\right)\right\rangle .
$$

This has an interesting consequence. If we inquire whether the EMI model determines a QFT the answer is yes, but with the expression of $I_{\mathrm{EMI}}(A, B)$ not corresponding to the mutual information, but rather to a correlation function of fluxes of GFF current. The Hilbert space and operator content can then be recovered in the usual way a QFT can be recovered from the correlation functions, and the result is the theory of the GFF current.

However, we have seen that the EMI cannot be interpreted as a MI for $d>2$. Recovering a QFT from the mutual information is very different (and more uncertain) than recovering it from correlation functions. For $d=2$, a free current can be recovered from the EMI interpreted as correlators, but, presumably, the fermion theory should be recovered interpreting it as a MI. The MI of a theory of a free current is a very different function from the one of the fermion [53].

Therefore, the Rényi twist operators cannot be coherent operators for $d>2$. For a free fermion theory, the twist fields can indeed be expressed as exponentials of fluxes of currents [22],

$$
\tau_{A}^{(n)}=e^{i \int_{\Sigma_{A}} \mathrm{~d} \sigma \eta_{\mu}\left(\sum_{k=-(n-1) / 2}^{(n-1) / 2} \frac{2 \pi k}{n} J_{(k)}^{\mu}\right)},
$$

where $J_{(k)}^{\mu}$ are mutually independent currents for free Dirac fields in the replica space. These currents are however not free fields for $d>2$; the expectation values of these operators give determinants and not exponentials of quadratic forms. This can be seen as the origin of the difference between the free fermion and the EMI model for $d>2$.

Pinching property. The expression (7.3) for the twist operators, with $J_{\mu}^{(n)}$ a generalized free field current, clashes directly with another expected property of the entropy. This is called the pinching property [7]. We have not listed it among the general properties of the entropy in the introduction because it expresses a relation of a property of the MI with properties of the QFT, rather than constraints on the MI function itself. This property is as follows.

Consider the null future horizon $H$ of the causal set $A$ corresponding to a sphere of radius $R$. This null surface is parametrized by the angular direction $\vec{\Omega}$ determining a null ray, and an affine parameter $s \in(0, R)$ along the null segments. We take $s=R$ as the position of the spherical base of the null surface and $s=0$ as the tip of the cone. Let us consider the causal region $A(\delta, \epsilon)$ determined by its future horizon:

$$
H-\left\{(\vec{\Omega}, s) /\left|\vec{\Omega}-\vec{\Omega}_{0}\right|<\epsilon, s>\delta\right\} .
$$

This results from cutting from $H$ a segment of a pencil of null generator around a fixed, but arbitrary, direction $\vec{\Omega}_{0}$. In the limit $\delta \rightarrow 0$, that is, when the cut on the null surface reaches the tip of the cone, the region $A(\delta, \epsilon)$ collapses to a null surface, and the causal region does not have any space-time volume. In the limit $\epsilon \rightarrow 0$, the region converges to 
the full spherical region. We consider the mutual information $I(A(\delta, \epsilon), B)$ for any fixed region $B$. The question is about the limit $\delta, \epsilon \rightarrow 0$ of this mutual information.

For interacting CFTs, including GFF, the correlation functions are too singular to obtain operators when smearing field operators on a null surface. Then the limit $\delta \rightarrow 0$ eliminates the algebra, and we have the pinching property [7]

$$
\lim _{\epsilon \rightarrow 0} \lim _{\delta \rightarrow 0} I(A(\delta, \epsilon), B)=0 .
$$

For a free theory - in the sense of being the theory of a field satisfying a local linear equation of motion, not a GFF - the algebra of operators can be localized on the null surface, and we have

$$
\lim _{\epsilon \rightarrow 0} \lim _{\delta \rightarrow 0} I(A(\delta, \epsilon), B)=I(A, B) .
$$

When an interacting theory has a free sector, the limit is non zero, but still

$$
\lim _{\epsilon \rightarrow 0} \lim _{\delta \rightarrow 0} I(A(\delta, \epsilon), B) \lesseqgtr I(A, B) .
$$

It is easy to see that the EMI model gives a non-pinching entropy function, satisfying eq. (7.8) just like the MI of free fields because the EMI is an integral over the boundary of the regions. The MI of a replicated theory is just the number of replicas times the mutual information of the theory. According to the ansatz (7.3) for the description of the twist operators of the EMI model, the algebra of this replicated EMI theory would contain a generalized free current. This must lead to discontinuities under pinching for this replicated model and hence contradicting the form of the mutual information of the EMI model. The only allowed operator content for continuity under pinching are free fields and their Wick polynomials.

New entropy inequalities? In this paper, we showed that the known properties of the entropy in QFT are not enough to allow for a complete description of the space of possible entropy functions. An important question is then what further properties are missing. A natural surmise is that there should be additional unknown inequalities. Indeed, operator correlations obey infinitely many inequalities and are precisely these inequalities the ones that allow for the reconstruction of the Hilbert space scalar product and the quantum theory. The Rényi entropies of integer order $n \geq 2$, as they are produced by expectation values of twist operators, also satisfy this infinite set of inequalities. In real time, these operator inequalities - equivalent to the reflection positive inequalities in imaginary time - are called wedge reflection positivity, Rindler positivity, or CRT positivity (for chargereflection-time inversion) $[10,54,55]$.

For the Rényi entropies, taking a series of regions $A_{i}, i=1, \cdots, m$, contained in the right wedge $x^{1}-\left|x^{0}\right|>0$, and defining the reflection $\bar{x}=\left(-x^{0},-x^{1}, x^{2}, \cdots, x^{d-1}\right)$ carrying the right wedge into the left one, we have

$$
\operatorname{det}\left(\left\{e^{(n-1) I_{n}\left(A_{i}, \bar{A}_{j}\right)}\right\}_{i, j=1}^{m}\right) \geq 0 .
$$

The $I_{n}(A, B)=S_{n}(A)+S_{n}(B)-S_{n}(A B)$ are the Rényi mutual informations. If we take all regions $A_{i}$ to be equal except for infinitesimal differences, we get an infinite series of 
inequalities involving derivatives of the Rényi entropies of arbitrarily large order. If we naively think in taking the limit $n \rightarrow 1$ in these inequalities we get an infinite set of polynomial inequalities for the mutual information

$$
\operatorname{det}\left(\left\{I\left(A_{i}, \bar{A}_{j}\right)+I\left(A_{i+1}, \bar{A}_{j+1}\right)-I\left(A_{i}, \bar{A}_{j+1}\right)-I\left(A_{i+1}, \bar{A}_{j}\right)\right\}_{i, j=1, \cdots, m-1}\right) \geq 0 .
$$

However, this set of inequalities for the entropy, does not hold in general theories and regions. An example is the holographic EE [54]. The reason is simply that it is not possible to analytically continue the inequalities down to $n=1$.

The present investigation suggests that inequalities generalizing the reflection positivity inequalities for operators is not the right idea to get the correct additional constraints. In fact, somewhat ironically, the EMI model, which obeys all the known constraints for a MI in QFT, and is not the MI of any QFT, also obeys the infinite series of inequalities (7.11). The reason is that the EMI comes from the expectation value of operators, as shown by eq. (7.4).

A natural expectation is that there are more inequalities following a different line, and generalizing strong subadditivity (SSA) rather than operator inequalities. In this same direction, it has recently been shown that SSA leads to unitarity bounds for fields with the tensor structure of free conformal primaries [7]. In this case, certain SSA inequalities are saturated for the conformal dimension at which the field becomes free. For fields with other tensor structures, it may well be the case that other inequalities generalizing SSA are needed to do the job of producing the unitarity bounds.

\section{Acknowledgments}

H.C. is indebted to Gonzalo Torroba for many useful insights on the subject of CFTs. This work was supported by the Simons foundation through the It From Qubit Simons collaboration. H.C. was also supported by CONICET, CNEA, and Universidad Nacional de Cuyo, Argentina.

\section{A EE universal coefficients for spheres and strips for the EMI}

From the results presented in section 3, we can extract two universal characteristic coefficients appearing in the entanglement entropy of the EMI model for general dimensions. These correspond, respectively, to the "strip coefficient" — characterizing entangling regions which are very large in all directions but one - and the one corresponding to spherical entangling surfaces.

Whenever we have an entangling region $A$ which is large in all directions, with a total area of $\mathcal{A}$ in such directions, and a small transversal dimension of length $r$, the entanglement entropy universal term takes the form

$$
\left.S_{\mathrm{EE}}\right|_{\text {univ }}=-k^{(d)} \frac{\mathcal{A}}{r^{d-1}}+\ldots
$$

where the dots denote subleading contributions. This coefficient is known analytically in general dimensions e.g., for holographic theories dual to Einstein gravity [12] and for 
free scalars and fermions [29] in terms of the two-dimensional entropic c-function $c(L) \equiv$ $L \cdot\left(\mathrm{d} S_{\mathrm{EE}} / \mathrm{d} L\right)$.

The coefficient $k^{(d)}$ can be extracted using the mutual information as follows. The idea is to consider two parallel entangling regions with the same shape as the one corresponding to the large dimensions of $A$ in the previous paragraph, separated a distance $r$. In that case, we have

$$
I=k^{(d)} \frac{\mathcal{A}}{r^{d-1}}+\ldots
$$

For the EMI model, we can then exploit the calculation for two concentric spheres presented in section 3 to obtain $k^{(d)}$. In order to do that, we take the final expression in eq. (3.48) for unboosted spheres and rewrite it in terms of the radii $R_{A}, R_{B}$. Then, we consider

$$
R_{A}=R-\delta / 2, \quad R_{B}=R+\delta / 2, \quad \delta \ll R,
$$

which puts the spheres very close to each other. Then, the result takes the form expected from eq. (A.2),

$$
I=k^{(d)} \frac{\mathcal{A}\left(\mathbb{S}^{d-2}\right)}{\delta^{d-1}}+\ldots \quad \text { where } \quad \mathcal{A}\left(\mathbb{S}^{d-2}\right) \equiv \frac{2 \pi^{\frac{d-1}{2}} R^{d-2}}{\Gamma\left(\frac{d-1}{2}\right)},
$$

is the area of the $\mathbb{S}^{d-2}$. Doing this, we find for the EMI

$$
k_{\mathrm{EMI}}^{(d)}=\frac{2 \pi^{\frac{d-2}{2}} \Gamma\left(\frac{d-2}{2}\right)}{\Gamma(d-2)} \kappa_{(d)} .
$$

When putting the two concentric spheres very close together, we are in practice using mutual information as a geometric regulator of entanglement entropy — see $[17,56]$ for more on this approach. Hence, in addition to the leading area-law-like piece in eq. (A.4), we can also extract the universal characteristic coefficients appearing in the logarithmic (even $d$ ) and constant (odd $d$ ) coefficients. In general we will have $\mathrm{e}^{11}$

$$
I=k^{(d)} \frac{\mathcal{A}}{r^{d-1}}+\cdots+2\left\{\begin{array}{lr}
(-1)^{\frac{d}{2}-1} 4 A^{(d)} \log (R / \delta) & (\text { even } d), \\
(-1)^{\frac{d-1}{2}} F^{(d)} & (\text { odd } d) .
\end{array}\right.
$$

In this expression, $A$ coincides with the trace-anomaly coefficient controlling the Euler density $E_{d}$ for even dimensional theories [58]

$$
\left\langle T_{a}^{a}\right\rangle=\sum_{i} B_{i} I_{i}-(-1)^{\frac{d}{2}} 2 A^{(d)} E_{d},
$$

and $(-1)^{\frac{d-1}{2}} F^{(d)}$ equals the free energy of the corresponding CFT when put on a round $\mathbb{S}^{d}[59]$.

For the EMI model, the expansion eq. (A.4) contains the terms expected from eq. (A.6) and we identify

$$
F_{\mathrm{EMI}}^{(d)}=\frac{2 \pi^{d-1}}{\Gamma(d-2)} \kappa_{(d)}, \quad A_{\mathrm{EMI}}^{(d)}=\frac{\pi^{d-2}}{\Gamma(d-2)} \kappa_{(d)} .
$$

\footnotetext{
${ }^{11}$ Our conventions agree with those of [57], for instance.
} 


\section{B OPE blocks and the EMI}

In this appendix, we are interested in studying the conformal block associated to a conserved current $J^{\mu}$ with $\Delta=d-1$ and $J=1$ and find in this the EMI formula for disjoint spheres. One can find parts of the material presented in this appendix in section 2 of [60] and section 3 of [61]. For a recent review of the shadow operator formalism and the original literature, see [62] and references therein.

In conformal field theories the set of quasiprimary operators and their descendents represents a complete basis of operators. Thus, when other operators are located away from points $x$ and 0 the product of say $\mathcal{O}_{i}(x)$ and $\mathcal{O}_{j}(0)$ inside an arbitrary correlator can be expressed as

$$
\mathcal{O}_{i}(x) \mathcal{O}_{j}(0)=\sum_{k} C_{i j k}|x|^{\Delta_{k}-\Delta_{i}-\Delta_{j}}\left(1+b_{1} x^{\mu} \partial_{\mu}+b_{2} x^{\mu} x^{\nu} \partial_{\mu} \partial_{\nu}+\cdots\right) \mathcal{O}_{k}(0),
$$

where $\Delta_{i}, \Delta_{j}$ and $\Delta_{k}$ are the scaling dimensions of their associated quasiprimary operators and the coefficients $C_{i j k}$ are dynamical parameters of the theory. We wrote the above expansion for scalar operators $\mathcal{O}_{i}, \mathcal{O}_{j}$ and $\mathcal{O}_{k}$, there are similar expansions for operators with arbitrary spin. In (B.1), the coefficients $b_{n}$ depend only on the $\Delta_{i}, \Delta_{j}, \Delta_{k}$ and thus are determined by the kinematics of the conformal symmetry. The contribution that comes from a particular primary and its descendents is known as the OPE block, and we denote it by

$$
\mathcal{B}_{k}^{i j}(x, 0)=|x|^{\Delta_{k}}\left(1+b_{1} x^{\mu} \partial_{\mu}+b_{2} x^{\mu} x^{\nu} \partial_{\mu} \partial_{\nu}+\cdots\right) \mathcal{O}_{k}(0) .
$$

In particular, $\mathcal{B}_{k}^{i j}(x, 0)$ becomes independent of $\Delta_{i}$ and $\Delta_{j}$ when $\mathcal{O}_{i}, \mathcal{O}_{j}$ have equal scaling dimension, $\Delta_{i}=\Delta_{j}$, thus in that case we denote it $\mathcal{B}_{k}(x, 0)$. Hereafter, we will restrict to this situation for simplicity.

In Lorentzian signature, when $x_{1}, x_{2}$ are time-like separated, one can use the following integral representation for the OPE block

$$
\mathcal{B}_{k}\left(x_{1}, x_{2}\right)=c_{k} \int_{D\left(x_{1}, x_{2}\right)} \mathrm{d}^{d} \xi\left(\frac{\left|x_{1}-\xi\right|\left|x_{2}-\xi\right|}{\left|x_{1}-x_{2}\right|}\right)^{\Delta_{k}-d} \mathcal{O}_{k}(\xi)
$$

This expression can be derived from the shadow operator formalism [60,62]. In (B.3), $c_{k}$ is an arbitrary constant to be determined, and the space-time integral is taken over the causal diamond whose future and past causal tips are $x_{1}$ and $x_{2}$ respectively and denote it as $D\left(x_{1}, x_{2}\right)$. This formula can be written in a more compact form, in terms of the conformal Killing vector $K^{\mu}$ that preserves the causal diamond as

$$
\mathcal{B}_{k}\left(x_{1}, x_{2}\right)=\frac{c_{k}}{(2 \pi)^{\Delta_{k}-d}} \int_{D\left(x_{1}, x_{2}\right)} \mathrm{d}^{d} \xi|K|^{\Delta_{k}-d} \mathcal{O}_{k}(\xi)
$$

where

$$
K^{\mu} \partial_{\mu}=-\frac{2 \pi}{\left(x_{1}-x_{2}\right)^{2}}\left[\left(x_{2}-\xi\right)^{2}\left(x_{1}^{\mu}-\xi^{\mu}\right)-\left(x_{1}-\xi\right)^{2}\left(x_{2}^{\mu}-\xi^{\mu}\right)\right] \partial_{\mu},
$$

and

$$
|K|=2 \pi \frac{\left|x_{1}-\xi\right|\left|x_{2}-\xi\right|}{\left|x_{1}-x_{2}\right|} .
$$


Indeed, (B.4) has a natural generalization for the OPE block of a spin $J$ symmetric quasiprimary operator $\mathcal{O}_{k}^{\mu_{1} \mu_{2} \cdots \mu_{J}}$ [61] which reads

$$
\mathcal{B}_{k, J}\left(x_{1}, x_{2}\right)=\frac{c_{k}}{(2 \pi)^{\Delta_{k}-d}} \int_{D\left(x_{1}, x_{2}\right)} \mathrm{d}^{d} \xi|K|^{\Delta_{k}-d-J} K^{\mu_{1}} \cdots K^{\mu_{J}} \mathcal{O}_{k, \mu_{1} \cdots \mu_{J}}(\xi) .
$$

The above expression can be further simplified for cases in which the operator $\mathcal{O}_{k, \mu_{1} \cdots \mu_{J}}(\xi)$ corresponds to a traceless conserved spin $J$ operator. In that case $\Delta_{k}=J+d-2$ and we can construct the conserved current $J_{\mu}$

$$
J_{\mu} \equiv K^{\mu_{2}} \cdots K^{\mu_{J}} \mathcal{O}_{k, \mu \mu_{2} \cdots \mu_{J}}(\xi)
$$

where conservation of $J_{\mu}$ follows from the fact that $K^{\mu}$ is a conformal Killing vector, and that $\mathcal{O}_{k, \mu \mu_{2} \cdots \mu_{J}}(\xi)$ is conserved and traceless. This results in the expression

$$
\mathcal{B}_{k, J}\left(x_{1}, x_{2}\right)=\frac{c_{k}}{(2 \pi)^{J-2}} \int_{D\left(x_{1}, x_{2}\right)} \mathrm{d}^{d} \xi \frac{K^{\mu} J_{\mu}}{|K|^{2}} .
$$

The space-time integral over the causal cone can be foliated with surfaces of constant $|K|$ so that $\mathrm{d}^{d} \xi=\mathrm{d}^{d-1} \Sigma|K| \mathrm{d} \lambda$, where $\mathrm{d}^{d-1} \Sigma$ is the area element induced on the constant $|K|$ surface and $\lambda$ is a flow parameter in the direction of $K^{\mu}$. In these coordinates, one realizes that the integral $\int \mathrm{d}^{d-1} \Sigma \ldots$ is independent of $\lambda$ (by the conservation equation) and thus, one gets an overall divergent factor $\int \mathrm{d} \lambda$ which can be absorbed into the coefficient $c_{k}$ by $c_{k} \rightarrow \tilde{c}_{k}$. Using the conservation of $J^{\mu}$ once more, one can show that the resulting integral over $\Sigma$ is indeed independent of the surface - it is not any more subject to be a constant $|K|$ surface; see [61] for a more detailed explanation on this. This results in

$$
\mathcal{B}_{k, J}\left(x_{1}, x_{2}\right)=\frac{\tilde{c}_{k}}{(2 \pi)^{J-2}} \int_{\Sigma_{A}} \mathrm{~d}^{d-1} \Sigma n_{A}^{\mu} J_{\mu}
$$

where $\Sigma_{A}$ is an arbitrary space-like surface with boundary on the sphere at the rim of the causal cone $\partial \Sigma_{A}=\mathbb{S}_{A}^{d-1}$. On the other hand, the conformal block associated to a spin $J$ symmetric operator can be rewritten as the following correlator

$$
G_{\Delta_{k}, J}^{d}(u, v)=\left\langle\mathcal{B}_{k, J}\left(x_{1}, x_{2}\right) \mathcal{B}_{k, J}\left(x_{3}, x_{4}\right)\right\rangle,
$$

in terms of the associated OPE blocks. This formula comes from the usual partial wave decomposition of a four point correlator and formulas (B.1) and (B.2). Thus, when such operator is conserved and traceless, one can use equation (B.10) in the above formula and get

$$
G_{d-2+J, J}^{d}(u, v)=\frac{\tilde{c}_{k}^{2}}{(2 \pi)^{2(J-2)}} \int_{\Sigma_{A}} \mathrm{~d}^{d-1} \Sigma_{A} n_{A}^{\mu} \int_{\Sigma_{B}} \mathrm{~d}^{d-1} \Sigma_{B} n_{B}^{\nu}\left\langle J_{\mu}(\xi) J_{\nu}\left(\xi^{\prime}\right)\right\rangle,
$$

where $J_{\mu}$ is given by (B.8). Furthermore, in the particular case of $J=1, J_{\mu}$ becomes a quasiprimary conserved current operator since $J_{\mu}=\mathcal{O}_{k, \mu}$, and its two-point function is fixed by conformal invariance to be

$$
\left\langle J_{\mu}(\xi) J_{\nu}\left(\xi^{\prime}\right)\right\rangle=\frac{1}{\left|\xi-\xi^{\prime}\right|^{2(d-1)}}\left(\eta_{\mu \nu}-\frac{2\left(\xi-\xi^{\prime}\right)_{\mu}\left(\xi-\xi^{\prime}\right)_{\nu}}{\left|\xi-\xi^{\prime}\right|^{2}}\right) .
$$


The above expression is obtained from the vector-vector correlator with $\Delta_{k}=d-1$. Further, (B.13) can be rewritten as

$$
\left\langle J_{\mu}(\xi) J_{\nu}\left(\xi^{\prime}\right)\right\rangle=-\frac{1}{2(d-1)(d-2)}\left(\partial_{\mu} \partial_{\nu}-g_{\mu \nu} \partial^{2}\right) \frac{1}{\left|\xi-\xi^{\prime}\right|^{2(d-2)}},
$$

where $g_{\mu \nu}$ is the Minkowski metric. Finally, plugging (B.14) into (B.12) results in a formula for the conformal block of a spin 1 conserved current that is identically proportional to the EMI formula as given in eq. (1.11).

\section{Conformal blocks for conserved currents}

The authors of [48] presented a general polynomial formula for the conformal blocks in any dimension as a function of its spin $J$ and conformal dimension $\Delta .{ }^{12}$ The expression has the form

$$
G_{\Delta, J}^{d}(u, v)=\sum_{n=0}^{\infty}|z|^{\Delta+n} \sum_{j} A_{n, j} \frac{C_{j}^{\frac{d-2}{2}}(\cos \varphi)}{C_{j}^{\frac{d-2}{2}}(1)}, \quad A_{n, j} \geq 0,
$$

where we expressed the $u$ and $v$ cross ratios in terms of the complex coordinates $z=|z| e^{i \varphi}$ with $\bar{z}=z^{*}$ via

$$
u \equiv z \bar{z}, \quad v \equiv(1-z)(1-\bar{z})
$$

$C_{j}^{\frac{d-2}{2}}(\cos \theta)$ are the Gegenbauer polynomials and the $j$ sum is over all the descendants of the primary operator $\Delta$ and thus it takes values

$$
j=J+n, J+n-2, \ldots, \max (J-n, J+n \bmod 2),
$$

for each $n$. The coefficients $A_{n, j}$ are some universal functions of $\Delta, J$ and $d$ that are fixed by conformal symmetry. These coefficients obey some recursion relations that can be solved explicitly [48]. In this section, we are interested in obtaining closed-form expressions for the simpler case in which the operator in question is a conserved current, and therefore its conformal dimension obeys

$$
\Delta=J+d-2, \quad J=0,1,2, \ldots
$$

In this case, it was found in [48], that only the maximal allowed $\operatorname{spin} j=J+n$ has a nonzero coefficient. Such coefficient is given by

$$
A_{n, J+n}=\frac{\left(J+\frac{d-2}{2}\right)_{n}(J+d-2)_{n}}{n !(2 J+d-2)_{n}},
$$

where $(x)_{n}=\Gamma(x+n) / \Gamma(x)$ are the Pochhammer coefficients. This fact simplifies considerably the formula (C.1), which reduces to

$$
G_{d-2+J, J}^{d}(u, v)=\sum_{n=0}^{\infty}|z|^{d-2+J+n} \frac{\left(J+\frac{d-2}{2}\right)_{n}(J+d-2)_{n}}{n !(2 J+d-2)_{n}} \frac{C_{J+n}^{\frac{d-2}{2}}(\cos \varphi)}{C_{J+n}^{\frac{d-2}{2}}(1)}
$$

\footnotetext{
${ }^{12}$ We thank Dalimil Mazáč for point out this reference to us.
} 
The angular variable is given by

$$
\cos \varphi=\frac{z+\bar{z}}{2|z|} .
$$

In particular, it is easy to check that for $\varphi=0$, which corresponds to the diagonal limit of the conformal blocks $(z=\bar{z})$ the above expression reduces to

$$
G_{d-2+J, J}^{d}(z)=z^{d-2+J}{ }_{2} F_{1}\left[J+\frac{d-2}{2}, J+d-2,2 J+d-2, z\right],
$$

where we identified the Hypergeometric function in the series expansion of (C.6). This expression is indeed equivalent to the general expression for the diagonal limit of conformal blocks found in [46] with $\Delta=J+d-2$.

We would like to write down a similar integral representation to the one we obtained for the $J=1$ conformal block. In this case, it would be convenient to use the following integral representation of the Gegenbauer polynomials

$$
C_{J+n}^{\frac{d-2}{2}}(\cos \varphi)=\frac{\Gamma(J+n+d-2)}{2^{d-3} n !\left(\Gamma\left(\frac{d-2}{2}\right)\right)^{2}} \int_{0}^{\pi} \mathrm{d} \theta \sin ^{d-3} \theta(\cos \varphi+i \sin \varphi \cos \theta)^{J+n} .
$$

The normalization coefficient is simply

$$
C_{J+n}^{\frac{d-2}{2}}(1)=\frac{\Gamma(J+n+d-2)}{2^{d-3} n !\left(\Gamma\left(\frac{d-2}{2}\right)\right)^{2}} \frac{\sqrt{\pi} \Gamma\left(\frac{d-2}{2}\right)}{\Gamma\left(\frac{d-1}{2}\right)} .
$$

Plugging this into (C.6) and interchanging the integral with the convergent sum, one gets

$$
\begin{aligned}
G_{d-2+J, J}^{d}(u, v)= & \frac{\Gamma\left(\frac{d-1}{2}\right)}{\sqrt{\pi} \Gamma\left(\frac{d-2}{2}\right)}|z|^{d-2} \int_{0}^{\pi} \mathrm{d} \theta \sin ^{d-3} \theta|z|^{J}(\cos \varphi+i \sin \varphi \cos \theta)^{J} \\
& \times \sum_{n=0}^{\infty}|z|^{n}(\cos \varphi+i \sin \varphi \cos \theta)^{n} \frac{\left(J+\frac{d-2}{2}\right)_{n}(J+d-2)_{n}}{n !(2 J+d-2)_{n}}
\end{aligned}
$$

where once again we recognize the sum over $n$ in the second line of the above equation as the series representation of a hypergeometric function, which leads to

$$
\begin{aligned}
G_{d-2+J, J}^{d}(u, v)= & \frac{2^{d-2} \Gamma\left(\frac{d-1}{2}\right)}{\sqrt{\pi} \Gamma\left(\frac{d-2}{2}\right)}|z|^{d-2} \int_{0}^{\pi / 2} \mathrm{~d} s \sin ^{d-3} s \cos ^{d-3} s\left(z \cos ^{2} s+\bar{z} \sin ^{2} s\right)^{J} \\
& \times{ }_{2} F_{1}\left[J+\frac{d-2}{2}, J+d-2,2 J+d-2 ; z \cos ^{2} s+\bar{z} \sin ^{2} s\right] .
\end{aligned}
$$

We have additionally re-expressed the integral in terms of $z$ and $\bar{z}$ and changed the integration variable $t \rightarrow s / 2$. Finally, we can get an integral with a bigger resemblance with (3.61) by doing $\cos s \rightarrow \xi$. In this way, we arrive at our final formula:

$$
\begin{aligned}
G_{d-2+J, J}^{d}(u, v)= & \frac{2^{d-2} \Gamma\left(\frac{d-1}{2}\right)}{\sqrt{\pi} \Gamma\left(\frac{d-2}{2}\right)}|z|^{d-2} \int_{0}^{1} \mathrm{~d} \xi\left(1-\xi^{2}\right)^{\frac{d-4}{2}} \xi^{d-3}\left(z \xi^{2}+\bar{z}\left(1-\xi^{2}\right)\right)^{J} \\
& \times{ }_{2} F_{1}\left[J+\frac{d-2}{2}, J+d-2,2 J+d-2 ; z \xi^{2}+\bar{z}\left(1-\xi^{2}\right)\right] . \quad(\mathrm{C} .1
\end{aligned}
$$


To express it in terms of $u$ and $v$, one would need to invert the relations (C.2) and plug them into the above formula. However, in these variables, the explicit formula is not particularly illuminating.

Finally, for $J=1$ the above expression becomes

$$
\begin{aligned}
G_{d-2+J, J}^{d}(u, v)= & \frac{2^{d-2} \Gamma\left(\frac{d-1}{2}\right)}{\sqrt{\pi} \Gamma\left(\frac{d-2}{2}\right)}|z|^{d-2} \int_{0}^{1} \mathrm{~d} \xi\left(1-\xi^{2}\right)^{\frac{d-4}{2}} \xi^{d-3}\left(z \xi^{2}+\bar{z}\left(1-\xi^{2}\right)\right) \\
& \times{ }_{2} F_{1}\left[\frac{d}{2}, d-1, d ; z \xi^{2}+\bar{z}\left(1-\xi^{2}\right)\right] .
\end{aligned}
$$

Unfortunately, it is not easy to analytically compare the above formula with (3.61). Nevertheless, we checked numerically that indeed (C.13) is equivalent to (3.61), when written in the same variables. This completes our checks of the formulas presented in section 3.1.3 particularly for $d=3$ and $d=5$.

\section{Coefficient of the first subleading term in the MI for a free fermion}

Here we want to compute the contribution of the two-copy primary $V_{\mu \nu}$ of eq. (6.4) to the mutual information of a free fermion. Using the signature $(-1,1, \cdots, 1)$ and normalizing the fermion field such that

$$
\langle\psi(0) \bar{\psi}(x)\rangle=i \frac{\not x}{|x|^{d}}
$$

the proportionality constant in the correlator (6.5) is $-4 d \operatorname{tr}(1)$.

The contribution to the mutual information is of the form

$$
\begin{aligned}
\Delta I(A, B) & =\gamma n_{A}^{\mu} n_{A}^{\nu}\left\langle V_{\mu \nu}(0) V_{\alpha \beta}(L \hat{l})\right\rangle n_{B}^{\alpha} n_{B}^{\beta} \\
& =-\frac{4 d \operatorname{tr}(1) \gamma}{L^{2 d}}\left(\left(2\left(n_{A} \cdot l\right)\left(n_{B} \cdot l\right)-\left(n_{A} \cdot n_{B}\right)\right)^{2}-\frac{1}{d}\right),
\end{aligned}
$$

where we seek to obtain the coefficient $\gamma$.

Following [7], this coefficient can be computed using the modular flow of spheres. We have

$$
\begin{aligned}
& \gamma n_{A}^{\alpha} n_{A}^{\alpha^{\prime}} n_{B}^{\beta} n_{B}^{\beta^{\prime}}\left\langle V_{\alpha \alpha^{\prime}}\left(r_{A}\right) V_{\delta \delta^{\prime}}\left(r_{1}\right)\right\rangle\left\langle V_{\beta \beta^{\prime}}\left(r_{B}\right) V_{\eta \eta^{\prime}}\left(r_{2}\right)\right\rangle \\
& =\int_{-\infty}^{\infty} \mathrm{d} s \frac{\pi}{4 \cosh ^{2}(\pi s)}\left\langle\left(\partial_{\delta} \bar{\psi}^{(s, A)}\right) \gamma_{\delta^{\prime}} \psi-\bar{\psi}^{(s, A)} \gamma_{\delta^{\prime}} \partial_{\delta} \psi+\left(\partial_{\delta} \bar{\psi}\right) \gamma_{\delta^{\prime}} \psi^{(s, A)}-\bar{\psi} \gamma_{\delta^{\prime}} \partial_{\delta} \psi^{(s, A)}\right\rangle\left(r_{1}\right) \\
& \quad \times\left\langle\left(\partial_{\eta} \bar{\psi}^{(s, B)}\right) \gamma_{\eta^{\prime}} \psi-\bar{\psi}^{(s, B)} \gamma_{\eta^{\prime}} \partial_{\eta} \psi+\left(\partial_{\eta} \bar{\psi}\right) \gamma_{\eta^{\prime}} \psi^{(s, B)}-\bar{\psi} \gamma_{\eta^{\prime}} \partial_{\eta} \psi^{(s, B)}\right\rangle\left(r_{2}\right), \quad r_{1,2}^{2} \rightarrow \infty .
\end{aligned}
$$

This formula gives the coefficient for any shape of $A, B$ in terms of the modular evolved fields $\psi^{(s, A)}, \psi^{(s, B)}$. For a sphere with $n$ in the time direction the modular flow is given by the conformal transformation (see [2])

$$
\begin{aligned}
y^{0}(x, \tau) & =N(\tau)^{-1} R\left(x^{0} R \cosh (2 \pi \tau)+\frac{1}{2}\left(R^{2}-x^{2}\right) \sinh (2 \pi \tau)\right), \\
y^{i}(x, \tau) & =N(\tau)^{-1} R^{2} x^{i} \\
N(\tau) & =x^{0} R \sinh (2 \pi \tau)+\frac{1}{2} \cosh (2 \pi \tau)\left(R^{2}-x^{2}\right)+\frac{1}{2}\left(R^{2}+x^{2}\right),
\end{aligned}
$$


with $x^{2}=-\left(x^{0}\right)^{2}+x^{i} x^{i}$. The modular evolved field is

$$
\psi^{(\tau, A)}(x)=\Omega^{(d-1) / 2}(x, \tau) S(\Lambda(x, \tau))^{-1} \psi(y(x, \tau)),
$$

where the Lorentz transformation $\Lambda$ and conformal factor $\Omega$ follow from

$$
\Lambda_{\nu}^{\mu}=\Omega^{-1} \frac{\mathrm{d} y^{\mu}}{\mathrm{d} x^{\nu}}
$$

$\Lambda$ is a Lorentz boost in the direction of $\vec{x}$ of a certain hyperbolic angle $\nu(\tau, x)$ given by

$$
\sinh (\nu(\tau, x))=-\frac{4 \sinh (\pi \tau)|\vec{x}|\left(R \cosh (\pi \tau)+x^{0} \sinh (\pi \tau)\right)}{R^{2}+2 R x^{0} \sinh (2 \pi \tau)+x^{2}+\left(R^{2}-x^{2}\right) \cosh (2 \pi \tau)} .
$$

The representation $S(\Lambda)$ can be obtained by the formula

$$
S=\cosh (\nu / 2) 1-\gamma^{0} \gamma^{i} \hat{x}_{i} \sinh (\nu / 2) .
$$

In formula (D.3) we have to choose a complex modular parameter for the transformation as

$$
\tau=s+i / 2
$$

and take $r_{1} \cdot n_{A}=r_{2} \cdot n_{B}=0$. We can use local coordinate systems for each sphere where $n_{A}, n_{B}$ are in the time direction for each of the correlators.

The right hand side of (D.3) gives

$$
\operatorname{tr}(1)^{2} \frac{2 \sqrt{\pi} d \Gamma(d+1)}{\Gamma\left(d+\frac{3}{2}\right)}\left(n_{A}^{\delta} n_{A}^{\delta^{\prime}}+\frac{1}{d} g^{\delta \delta^{\prime}}\right)\left(n_{B}^{\eta} n_{B}^{\eta^{\prime}}+\frac{1}{d} g^{\eta \eta^{\prime}}\right) \frac{R_{A}^{d} R_{B}^{d}}{r_{1}^{2 d} r_{2}^{2 d}} .
$$

From which we obtain the coefficient

$$
\gamma=\frac{\sqrt{\pi} \Gamma(d+1)}{8 d \Gamma\left(d+\frac{3}{2}\right)} R_{A}^{d} R_{B}^{d}
$$

Upon replacing in (D.2), the coefficient of the contribution (6.9) in the main text follows.

Open Access. This article is distributed under the terms of the Creative Commons Attribution License (CC-BY 4.0), which permits any use, distribution and reproduction in any medium, provided the original author(s) and source are credited.

\section{References}

[1] R.F. Streater and A.S. Wightman, PCT, spin and statistics and all that, Princeton University Press, Princeton, NJ, U.S.A. (2000).

[2] R. Haag, Local quantum physics: fields, particles, algebras, Springer, Berlin, Heidelberg, Germany (1996).

[3] J. Cardy, Some results on the mutual information of disjoint regions in higher dimensions, J. Phys. A 46 (2013) 285402 [arXiv: 1304.7985] [InSPIRE]. 
[4] C. Agón and T. Faulkner, Quantum corrections to holographic mutual information, JHEP 08 (2016) 118 [arXiv: 1511.07462] [InSPIRE].

[5] C. Agón, P. Bueno and H. Casini, to appear.

[6] M. Ohya and D. Petz, Quantum entropy and its use, Springer, Berlin, Heidelberg, Germany (1993).

[7] H. Casini, E. Testé and G. Torroba, Mutual information superadditivity and unitarity bounds, arXiv:2103.15847 [INSPIRE].

[8] H. Casini, E. Testé and G. Torroba, Modular Hamiltonians on the null plane and the Markov property of the vacuum state, J. Phys. A 50 (2017) 364001 [arXiv:1703.10656] [InSPIRE].

[9] H. Casini, E. Testé and G. Torroba, All the entropies on the light-cone, JHEP 05 (2018) 005 [arXiv: 1802.04278] [INSPIRE].

[10] H. Casini, Entropy inequalities from reflection positivity, J. Stat. Mech. 1008 (2010) P08019 [arXiv: 1004.4599] [INSPIRE].

[11] S. Ryu and T. Takayanagi, Holographic derivation of entanglement entropy from AdS/CFT, Phys. Rev. Lett. 96 (2006) 181602 [hep-th/0603001] [inSPIRE].

[12] S. Ryu and T. Takayanagi, Aspects of holographic entanglement entropy, JHEP 08 (2006) 045 [hep-th/0605073] [INSPIRE].

[13] V.E. Hubeny, M. Rangamani and T. Takayanagi, A covariant holographic entanglement entropy proposal, JHEP 07 (2007) 062 [arXiv:0705.0016] [INSPIRE].

[14] A. Lewkowycz and J. Maldacena, Generalized gravitational entropy, JHEP 08 (2013) 090 [arXiv: 1304.4926] [INSPIRE].

[15] T. Faulkner, A. Lewkowycz and J. Maldacena, Quantum corrections to holographic entanglement entropy, JHEP 11 (2013) 074 [arXiv: 1307.2892] [INSPIRE].

[16] H. Casini and M. Huerta, Remarks on the entanglement entropy for disconnected regions, JHEP 03 (2009) 048 [arXiv:0812.1773] [INSPIRE].

[17] H. Casini, M. Huerta, R.C. Myers and A. Yale, Mutual information and the F-theorem, JHEP 10 (2015) 003 [arXiv:1506.06195] [INSPIRE].

[18] P. Bueno, R.C. Myers and W. Witczak-Krempa, Universality of corner entanglement in conformal field theories, Phys. Rev. Lett. 115 (2015) 021602 [arXiv:1505.04804] [INSPIRE].

[19] W. Witczak-Krempa, L.E. Hayward Sierens and R.G. Melko, Cornering gapless quantum states via their torus entanglement, Phys. Rev. Lett. 118 (2017) 077202 [arXiv: 1603.02684] [INSPIRE].

[20] P. Bueno, H. Casini and W. Witczak-Krempa, Generalizing the entanglement entropy of singular regions in conformal field theories, JHEP 08 (2019) 069 [arXiv:1904.11495] [INSPIRE].

[21] B. Estienne, J.-M. Stéphan and W. Witczak-Krempa, Cornering the universal shape of fluctuations, arXiv:2102.06223 [INSPIRE].

[22] H. Casini, C.D. Fosco and M. Huerta, Entanglement and alpha entropies for a massive Dirac field in two dimensions, J. Stat. Mech. 0507 (2005) P07007 [cond-mat/0505563] [INSPIRE].

[23] B. Swingle, Mutual information and the structure of entanglement in quantum field theory, arXiv: 1010.4038 [INSPIRE]. 
[24] P.D. Hislop and R. Longo, Modular structure of the local algebras associated with the free massless scalar field theory, Commun. Math. Phys. 84 (1982) 71 [INSPIRE].

[25] B. Chen, L. Chen, P.-X. Hao and J. Long, On the mutual information in conformal field theory, JHEP 06 (2017) 096 [arXiv: 1704.03692] [INSPIRE].

[26] D.M. Hofman and J. Maldacena, Conformal collider physics: energy and charge correlations, JHEP 05 (2008) 012 [arXiv:0803.1467] [INSPIRE].

[27] D.M. Hofman, D. Li, D. Meltzer, D. Poland and F. Rejon-Barrera, A proof of the conformal collider bounds, JHEP 06 (2016) 111 [arXiv: 1603.03771] [INSPIRE].

[28] H. Casini and M. Huerta, Reduced density matrix and internal dynamics for multicomponent regions, Class. Quant. Grav. 26 (2009) 185005 [arXiv:0903.5284] [INSPIRE].

[29] H. Casini and M. Huerta, Entanglement entropy in free quantum field theory, J. Phys. A 42 (2009) 504007 [arXiv:0905.2562] [INSPIRE].

[30] I.R. Klebanov, S.S. Pufu and B.R. Safdi, F-theorem without supersymmetry, JHEP 10 (2011) 038 [arXiv: 1105.4598] [INSPIRE].

[31] M. Mariño, Lectures on localization and matrix models in supersymmetric Chern-Simons-matter theories, J. Phys. A 44 (2011) 463001 [arXiv:1104.0783] [INSPIRE].

[32] H. Casini and M. Huerta, Universal terms for the entanglement entropy in $2+1$ dimensions, Nucl. Phys. B 764 (2007) 183 [hep-th/0606256] [INSPIRE].

[33] T. Hirata and T. Takayanagi, AdS/CFT and strong subadditivity of entanglement entropy, JHEP 02 (2007) 042 [hep-th/0608213] [INSPIRE].

[34] B. Swingle, Mutual information and the structure of entanglement in quantum field theory,

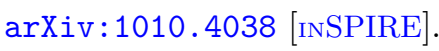

[35] H. Osborn and A.C. Petkou, Implications of conformal invariance in field theories for general dimensions, Annals Phys. 231 (1994) 311 [hep-th/9307010] [INSPIRE].

[36] T. Faulkner, R.G. Leigh and O. Parrikar, Shape dependence of entanglement entropy in conformal field theories, JHEP 04 (2016) 088 [arXiv:1511.05179] [INSPIRE].

[37] S.N. Solodukhin, Entanglement entropy, conformal invariance and extrinsic geometry, Phys. Lett. B 665 (2008) 305 [arXiv:0802.3117] [INSPIRE].

[38] J.S. Dowker and R. Critchley, The stress tensor conformal anomaly for scalar and spinor fields, Phys. Rev. D 16 (1977) 3390 [INSPIRE].

[39] M.J. Duff, Observations on conformal anomalies, Nucl. Phys. B 125 (1977) 334 [InSPIRE].

[40] F. Bastianelli, S. Frolov and A.A. Tseytlin, Conformal anomaly of $(2,0)$ tensor multiplet in six-dimensions and AdS/CFT correspondence, JHEP 02 (2000) 013 [hep-th/0001041] [INSPIRE].

[41] B.R. Safdi, Exact and numerical results on entanglement entropy in $(5+1)$-dimensional CFT, JHEP 12 (2012) 005 [arXiv:1206.5025] [INSPIRE].

[42] J. Long, On co-dimension two defect operators, arXiv:1611.02485 [INSPIRE].

[43] B. Chen and J. Long, Rényi mutual information for a free scalar field in even dimensions, Phys. Rev. D 96 (2017) 045006 [arXiv:1612.00114] [InSPIRE].

[44] B. Chen, L. Chen, P.-X. Hao and J. Long, On the mutual information in conformal field theory, JHEP 06 (2017) 096 [arXiv: 1704.03692] [INSPIRE]. 
[45] F.A. Dolan and H. Osborn, Conformal partial waves: further mathematical results, arXiv:1108.6194 [INSPIRE].

[46] M. Hogervorst, H. Osborn and S. Rychkov, Diagonal limit for conformal blocks in d dimensions, JHEP 08 (2013) 014 [arXiv: 1305.1321] [INSPIRE].

[47] S. El-Showk, M.F. Paulos, D. Poland, S. Rychkov, D. Simmons-Duffin and A. Vichi, Solving the 3d Ising model with the conformal bootstrap, Phys. Rev. D 86 (2012) 025022 [arXiv: 1203.6064] [INSPIRE].

[48] M. Hogervorst and S. Rychkov, Radial coordinates for conformal blocks, Phys. Rev. D 87 (2013) 106004 [arXiv: 1303.1111] [INSPIRE].

[49] F.A. Dolan and H. Osborn, Conformal four point functions and the operator product expansion, Nucl. Phys. B 599 (2001) 459 [hep-th/0011040] [INSPIRE].

[50] N. Shiba, Direct calculation of mutual information of distant regions, JHEP 09 (2020) 182 [arXiv: 1907.07155] [INSPIRE].

[51] H. Epstein, On the Borchers class of a free field, Nuovo Cim. 27 (1963) 886.

[52] H. Casini and M. Huerta, Entanglement entropy of a Maxwell field on the sphere, Phys. Rev. D 93 (2016) 105031 [arXiv: 1512.06182] [INSPIRE].

[53] R.E. Arias, H. Casini, M. Huerta and D. Pontello, Entropy and modular Hamiltonian for a free chiral scalar in two intervals, Phys. Rev. D 98 (2018) 125008 [arXiv:1809.00026] [INSPIRE].

[54] H. Casini and M. Huerta, Positivity, entanglement entropy, and minimal surfaces, JHEP 11 (2012) 087 [arXiv: 1203.4007] [INSPIRE].

[55] D. Blanco, L. Lanosa, M. Leston and G. Pérez-Nadal, Rényi mutual information inequalities from Rindler positivity, JHEP 12 (2019) 078 [arXiv: 1909.03144] [INSPIRE].

[56] H. Casini, Mutual information challenges entropy bounds, Class. Quant. Grav. 24 (2007) 1293 [gr-qc/0609126] [INSPIRE].

[57] R.C. Myers and A. Sinha, Seeing a c-theorem with holography, Phys. Rev. D 82 (2010) 046006 [arXiv: 1006.1263] [INSPIRE].

[58] M.J. Duff, Twenty years of the Weyl anomaly, Class. Quant. Grav. 11 (1994) 1387 [hep-th/9308075] [INSPIRE].

[59] H. Casini, M. Huerta and R.C. Myers, Towards a derivation of holographic entanglement entropy, JHEP 05 (2011) 036 [arXiv:1102.0440] [INSPIRE].

[60] B. Czech, L. Lamprou, S. McCandlish, B. Mosk and J. Sully, A stereoscopic look into the bulk, JHEP 07 (2016) 129 [arXiv:1604.03110] [INSPIRE].

[61] J. de Boer, F.M. Haehl, M.P. Heller and R.C. Myers, Entanglement, holography and causal diamonds, JHEP 08 (2016) 162 [arXiv: 1606. 03307] [INSPIRE].

[62] D. Simmons-Duffin, Projectors, shadows, and conformal blocks, JHEP 04 (2014) 146 [arXiv: 1204.3894] [INSPIRE]. 